\title{
A role for mDia, a Rho-regulated actin nucleator, in tangential migration of interneuron precursors.
}

\section{$\operatorname{AUTHOR}(\mathrm{S})$ :}

Shinohara, Ryota; Thumkeo, Dean; Kamijo, Hiroshi; Kaneko, Naoko; Sawamoto, Kazunobu; Watanabe, Keisuke; Takebayashi, Hirohide; ... Ishizaki, Toshimasa; Furuyashiki, Tomoyuki; Narumiya, Shuh

\section{CITATION:}

Shinohara, Ryota ... [et al]. A role for mDia, a Rho-regulated actin nucleator, in tangential migration of interneuron precursors.. Nature neuroscience 2012, 15(3): 373-380

\section{ISSUE DATE:}

2012-01-15

\section{URL:}

http://hdl.handle.net/2433/152361

\section{RIGHT:}

(C) 2012 Nature America, Inc.; 許諾条件により本文は2012-07-15に公開.; この論文は出版社版でありません。引用の際には出版社版をご確認ご 利用ください。; This is not the published version. Please cite only the published version. 


\section{A role for mDia, a Rho-regulated actin nucleator, in tangential migration of interneuron precursors}

Ryota Shinohara ${ }^{1}$, Dean Thumkeo ${ }^{1}$, Hiroshi Kamijo ${ }^{1}$, Naoko Kaneko ${ }^{2}$, Kazunobu Sawamoto $^{2}$, Keisuke Watanabe ${ }^{3,6}$, Hirohide Takebayashi ${ }^{3,6}$, Hiroshi Kiyonari ${ }^{4}$, Toshimasa Ishizaki ${ }^{1}$, Tomoyuki Furuyashiki ${ }^{1,5}$, and Shuh Narumiya ${ }^{1,5^{*}}$

${ }^{1}$ Department of Pharmacology, Kyoto University Graduate School of Medicine, Kyoto 606-8501, Japan

${ }^{2}$ Department of Developmental and Regenerative Biology, Institute of Molecular Medicine, Nagoya City University Graduate School of Medical Sciences, Nagoya 467-8601, Japan

${ }^{3}$ Department of Morphological Neural Science, Graduate School of Medical Science, Kumamoto University, Kumamoto 860-8556, Japan

${ }^{4}$ Laboratory for Animal Resources and Genetic Engineering, RIKEN Center for Developmental Biology, Kobe 650-0047, Japan

${ }^{5}$ Core Research for Evolutional Science and Technology, Tokyo 102-0075, Japan

${ }^{6}$ Present address: Division of Neurobiology and Anatomy, Graduate School of Medical and Dental Sciences, Niigata University, Niigata 951-8510, Japan

*Corresponding author: Department of Pharmacology, Kyoto University Graduate School of Medicine, Kyoto 606-8501, Japan.

Tel: +81-75-753-4392

Fax: +81-75-753-4693

Email: snaru@mfour.med.kyoto-u.ac.jp 


\section{ABSTRACT}

In brain development, excitatory and inhibitory neurons show distinct types of migration, radial migration and tangential migration, respectively. Whether these two types of migration are operated by similar cellular mechanisms remains unclear. Here we examined mice deficient in mDia, a Rho-regulated actin nucleator, in neuronal migration. mDia deficiency impaired tangential migration of cortical and olfactory inhibitory interneurons, whereas radial migration and consequent layer formation of cortical excitatory neurons were unaffected. mDia-deficient neuroblasts exhibited reduced separation of the centrosome from the nucleus and retarded nuclear translocation. Concomitantly, anterograde F-actin movement and the following rear F-actin condensation that occur during centrosomal and nuclear movement of wild-type cells, respectively, were impaired in mDia-deficient neuroblasts. Blockade of ROCK, another Rho effector regulating myosin II, also impaired nuclear translocation. These results suggest that the Rho-mDia/ROCK signaling critically regulates nuclear translocation via F-actin dynamics in tangential migration, while this mechanism is dispensable in radial migration. 


\section{INTRODUCTION}

Migration of neuronal precursors determines cellular architecture of the brain for subsequent neural network formation. There are two types of neuronal migration ${ }^{1-3}$. Precursors of excitatory, glutamatergic neurons born in the cortical ventricular zone radially migrate towards the pial surface as successive waves to form cortical layers. On the other hand, precursors of GABAergic interneurons born in the ganglionic eminences (GEs) tangentially migrate for a long distance to cortical and subcortical regions. A subset of tangentially migrating precursors remains to be generated after development in the subventricular zone (SVZ) located on the lateral wall of the lateral ventricle ${ }^{4}$. These precursors called SVZ neuroblasts migrate towards the olfactory bulb through the rostral migratory stream (RMS), and become differentiated to olfactory bulb interneurons for renewal ${ }^{5}$.

Neuronal precursors accomplish migration through a repetitive cycle composed of a growth of the leading process and saltatory movement of the nucleus called nuclear translocation ${ }^{1,3,6,7}$. Before nuclear translocation, the centrosome moves forward to a "swelling" that is transiently formed at a portion of the leading process proximal to the cell body, and then the nucleus moves towards the centrosome. Since microtubules connect the nucleus to the centrosome, many studies analyzed roles of microtubules in nuclear translocation, and it has been proposed that microtubules and dynein-mediated motor activity provide the major force pulling the nucleus towards the centrosome ${ }^{8-10}$.

However, most of these studies were performed on radial migration, and whether radial migration and tangential migration are operated by similar or distinct mechanisms remains unknown. Tangential migration is typically much faster than radial migration, and cortical and olfactory interneuron precursors exhibit much larger saltatory 
movement of the nucleus to the centrosome $e^{3,11-13}$. Notably, previous studies suggested that microtubules may not be critical for nuclear translocation in migration of cortical and olfactory interneuron precursors in vitro ${ }^{14-16}$. These findings suggest a possibility that contribution of microtubules is different between radial and tangential migration.

In addition to microtubules, recent studies have begun to reveal involvement of actin cytoskeleton in neuronal migration. Pharmacological blockade of myosin II activity halts nuclear translocation in both radial migration ${ }^{10}$ and tangential migration $^{14,16,17}$. Since myosin II and its phosphorylated form are enriched at the cell rear undergoing nuclear translocation of interneuron precursors, it has been suggested that actomyosin-based contractility pushes the nucleus from the rear during nuclear translocation in tangential migration. The Rho family of small GTPases such as Rho, Rac and Cdc42 is critical for reorganization of actin cytoskeleton in various cell types including neurons ${ }^{18,19}$. Previous studies suggested that the Rac1/Cdc42 pathway promotes, while attenuation of RhoA is required for, radial migration ${ }^{6,20,21}$. However, little is known on roles of Rho GTPases in tangential migration. Furthermore, how Rho signaling is linked to the action of actomyosin in neuronal migration remains unknown. Among the Rho family members, Rho regulates actomyosin-based contractility through binding to ROCK (Rho-associated kinase) and $\mathrm{mDia}^{22}$. ROCK facilitates phosphorylation and activation of myosin light chain to induce myosin-based contractility ${ }^{23,24}$. On the other hand, mDia nucleates actin oligomer and facilitates actin polymerization to produce long straight actin filaments ${ }^{25-27}$. In non-neuronal cultured cells, cooperation between mDia and ROCK is required to properly reproduce the dense and parallel pattern of Rho-induced actin stress fibers ${ }^{25}$. Here we have generated mice lacking mDia1 and mDia3, two isoforms expressed in the brain, in combination, and 
found that mDia-mediated actin dynamics is critical for tangential migration of inhibitory interneuron precursors, but is dispensable for radial migration. 


\section{RESULTS}

\section{A role of $\mathrm{mDia}$ in migration of interneuron precursors}

We examined expression of mDia isoforms in developing and adult brains. mDia1 and mDia3 are present in developing and adult brains, and their mRNA is expressed in various brain regions including the GEs and adult SVZ (Supplementary Figs. 1-3). To analyze mDia functions in vivo, we generated mice lacking either mDia1 or mDia3. Given that these mice appear to develop normally, we generated mice lacking both isoforms in combination (mDia-DKO), and examined mDia functions in brain development (Supplementary Figs. 4-5).

We asked whether mDia deficiency affects brain organization. Nissl staining revealed that supragranular (layer II/III), granular (layer IV), and infragranular (layer $\mathrm{V} / \mathrm{VI}$ ) layers in the somatosensory cortex were formed in adult mDia-DKO mice as clearly as wild-type mice (Supplementary Fig. 6a). Furthermore, immunofluorescence for Cux1, Ctip2, and Foxp2, markers for cortical layer II-IV, V/VI, and VI, respectively, showed normal organization of respective layers in adult mDia-DKO mice (Supplementary Fig. 6b-d). Distribution of Cux1-positive cells was normal in the cortex of mDia-DKO mice at E16.5 and P0 (Supplementary Fig. 7). These data suggest that the layer formation of cortical excitatory neurons by radial migration is not impaired in mDia-DKO mice. To confirm that radial migration properly occurs in mDia-DKO brain, we injected 5-ethynyl-2'-deoxyuridine (EdU) into pregnant mice carrying embryos of E15.5, and examined migration of EdU-labelled cells. In both wild-type and mDia DKO embryos, EdU-labelled cells migrate from the cortical ventricular zone toward the pial surface, and at E18.5, more EdU-labeled cells reached the cortical surface in mDia-DKO embryos compared to wild-type embryos (Fig. 1), 
indicating that mDia deficiency did not impair, but promote, radial migration.

In contrast, we noted that significantly fewer GAD65/67-positive cells entered the pallium and reached the top of the cerebral cortex in mDia-DKO embryos at E14.5 than in wild-type embryos (Fig. 2a,b), suggesting impaired tangential migration of cortical inhibitory interneuon precursors. Consistently, the number of GABA-containing cells was reduced in the cerebral cortex of mDia-DKO embryos at E16.5 compared to control littermates (Supplementary Fig. 7a). Further, the number of parvalbumin-positive interneurons was reduced in the motor cortex and hippocampus of adult mDia-DKO mice compared to wild-type and mDia3-KO mice (Fig. 2c,d). The reduction was not significant in the somatosensory cortex, which is located closer to the MGE than the above two regions (Fig. 2d). These observations suggest that the migration deficit of interneuron precursors is more evident for those traveling a longer distance of tangential migration in mDia-DKO mice.

Given that olfactory interneurons are continuously replaced by SVZ neuroblasts that migrate through the $\mathrm{RMS}^{4}$, we examined their migration in adult mDia-DKO mice by doublecortin immunostaining. Doublecortin-positive SVZ neuroblasts abnormally accumulated at the posterior level of the RMS (pRMS), while they were much fewer at the anterior level of the RMS (aRMS) and the olfactory bulb, of mDia-DKO mice (Fig. 3a,b and Supplementary Fig. 8a). Consistently, though the size of overall brain appears to be unaffected, the size of the olfactory bulb was reduced with marked loss of granule cells in adult mDia-DKO mice (Fig. 3c). Consistent with the fact that the granule cells expand after birth, mDia deficiency affected neither the size of the olfactory bulb nor the formation of the mitral cell layer at E18.5 (Supplementary Fig.9). In the olfactory bulb of adult mDia-DKO mice, Reelin-positive mitral cells were 
present in comparable numbers to wild-type mice, but the density of these cells was increased (Supplementary Fig. 8b,c).

Notably, migration of SVZ neuroblasts was already disorganized before neuroblasts entered the RMS. Doublecortin-positive neuroblasts in adult wild-type mice formed uninterrupted migratory chains on the lateral ventricle wall, which run parallel to the dorsal ridge of the lateral ventricle (Supplementary Fig. 10a). In adult mDia-DKO mice, these parallel bundles were almost absent, and the migratory chains were fragmented and dispersed (Supplementary Fig. 10a). Further, elongation of nuclei, which typically occurs during nuclear translocation ${ }^{10}$, was seen in wild-type and mDia3-KO neuroblasts but not in mDia-DKO neuroblasts (Supplementary Fig. 10b,c).

To exclude a possibility that impaired cell proliferation causes reduction in cortical and olfactory interneurons, we pulse-labelled E13.5 embryos and adult mice with EdU, and examined EdU incorporation into neural stem/progenitor cells one hour after injection. mDia-DKO mice showed normal extent and distribution of EdU-labeled cells in LGE, MGE and adult SVZ (Supplementary Fig. 11), suggesting that mDia deficiency does not affect proliferation of neural stem/progenitor cells.

To test whether the abnormality in migration of cortical and olfactory interneuron precursors in mDia-DKO mice is attributable to an intrinsic deficit of neuronal precursors, we examined migration of these cells in vitro. To this end, isolated explants of E14.5 MGE were cultured in Matrigel. Whereas wild-type and mDia3-KO MGE cells migrated from the explants, a fewer number of mDia-DKO MGE cells migrated out of the explants (Fig. 4a,b). We similarly examined migration of mDia-DKO neuroblasts by culture of SVZ explant in Matrigel, in which SVZ neuroblasts migrate in chain $^{12}$. Many neuroblasts migrated from wild-type and mDia-3KO SVZ explants in 
chains as seen in vivo (Fig. 4c-e and Supplementary Video 1). In contrast, migration of SVZ neuroblasts from mDia-DKO explants was reduced as observed in MGE cells, and little chain formation was seen (Fig. 4c-e and Supplementary Video 2). These findings indicate that mDia-deficient interneuron precursors are impaired in their migration per se.

\section{A role of $\mathrm{mDia}$ in nuclear translocation during migration}

Given that SVZ neuroblasts are more tractable for genetic manipulation by in utero electroporation than MGE cells, we used SVZ neuroblasts as an in vitro model for tangential migration. To characterize defective migration of mDia-DKO neuroblasts, we introduced an EGFP-expressing vector into SVZ at E16.5 by in utero electroporation. After 24 hours, we observed migration of EGFP-expressing SVZ neuroblasts in Matrigel by time-lapse fluorescent microscopy. Wild-type neuroblasts exhibited polarized morphology and migrated with cyclic motions; the growth of a leading process and subsequent nuclear translocation (Fig. 5a,b and Supplementary Video 3). mDia-DKO neuroblasts exhibited polarized morphology like wild-type neuroblasts, and the length of a leading and trailing process and the number of processes and branches were similar between the two genotypes (Fig. 5a,c and Supplementary Fig. 12). In contrast, the motility of the cell body was slowed in mDia-DKO neuroblasts (Fig. 5a,b,d and Supplementary Video 4).

Since the centrosome is thought to mediate nuclear translocation in radial migration, we visualized the centrosome with monomeric Kusabira Orange-fused PACT (pericentrin-AKAP450 centrosomal targeting) domain (PACT-mKO1) ${ }^{28}$ introduced simultaneously with EGFP. In wild-type neuroblasts $(0 \mathrm{~min}$ in Fig. $6 \mathbf{6 a}$ and 
Supplementary Video 5), a swelling first appeared ahead of the cell body, and the centrosome was typically observed in the swelling (38/49 events of nuclear translocation). The swelling then proceeded with the centrosome away from the cell body, concomitantly with rapid extension of the leading process (3 and 6 min in Fig. 6a). Subsequently, the cell body proceeded and eventually fused with the swelling (6 and 9 min in Fig. 6a). In mDia-DKO neuroblasts, although the swelling was formed and the centrosome was observed in the swelling at a similar frequency to wild-type cells (20/24 events of nuclear translocation), the swelling and the centrosome were less separated from the cell body than in wild-type neuroblasts (Fig. 6a and Supplementary Video 6). Thus, the maximum distance between the centrosome and the cell body prior to nuclear translocation was significantly reduced in mDia-DKO neuroblasts (wild-type, $13.90 \pm 1.80 \mu \mathrm{m}, n=27$ cells; mDia-DKO, $7.93 \pm 0.88 \mu \mathrm{m}, n=18$ cells; $P<0.05$, $t$-test). The maximum velocity of centrosomal movement (and thus movement of the swelling) before nuclear translocation was also slowed (Fig. 6b; wild-type, $2.81 \pm 0.25$ $\mu \mathrm{m} \min ^{-1}, n=30$ cells; mDia-DKO, $1.87 \pm 0.26 \mu \mathrm{m} \mathrm{min}^{-1}, n=23$ cells; $P<0.05$, $t$-test), and the centrosome also fluctuated within the swelling in mDia-DKO neuroblasts (Fig. 6a). Notably, leading process motility that was synchronized with centrosomal movement was also impeded (Fig. 6a), as indicated by its averaged velocity during the observation period (wild-type, $1.40 \pm 0.13 \mu \mathrm{m} \mathrm{min}^{-1}, n=29$ cells; mDia-DKO, $0.85 \pm$ $0.07 \mu \mathrm{m} \min ^{-1}, n=22$ cells; $P<0.01, t$-test). Although the swelling remained close to the cell body in mDia-DKO neuroblasts, the cell body remained unfused with the swelling for longer duration (wild-type, $12.34 \pm 1.62 \mathrm{~min}, n=25$ cells; mDia-DKO, $23.02 \pm 4.68 \mathrm{~min}, n=16$ cells; $P<0.05$, $t$-test). Consequently, the maximum velocity of 
movement of the cell body was reduced (Fig. 6b; wild-type, $3.53 \pm 0.37 \mu \mathrm{m} \mathrm{min}^{-1}, n=$ 30 cells; mDia-DKO, $1.96 \pm 0.39 \mu \mathrm{m} \mathrm{min}^{-1}, n=23$ cells; $P<0.01, t$-test). These results indicate that mDia is critical for both separation of the centrosome from the nucleus and nuclear translocation in migration of SVZ neuroblasts.

\section{mDia mediates F-actin dynamics for nuclear translocation}

Previous studies reported that microtubules may not be critical for nuclear translocation in cortical and olfactory inhibitory interneuron precursors ${ }^{14-16}$. Nonetheless, since mDia can bind to microtubule-associated proteins, EB1 and $\mathrm{APC}^{29}$, and has been implicated in microtubule reorientation and stabilization in non-neuronal cells ${ }^{30,31}$, we examined microtubule structure and stability in mDia-DKO neuroblasts. Immunofluorescence for $\alpha$-tubulin revealed that 17 out of 17 mDia-DKO neuroblasts exhibited cage-like microtubule structure around the nucleus as seen in wild-type cells (Supplementary Fig. 13a). Tubulin acetylation, a posttranslational modification which occurs in stable and long-lived microtubules ${ }^{32}$, was observed in this cage-like microtubule structure, and was not apparently disturbed in mDia-DKO neuroblasts (Supplementary Fig. 13b). These results indicate that the migration deficit in these mutant cells is not due to change in microtubules, although we do not exclude possible involvement of microtubules in migration of SVZ neuroblasts.

Given that mDia is an actin nucleator ${ }^{26,27}$, we examined whether F-actin dynamics was altered in mDia-DKO neuroblasts by visualizing F-actin dynamics with Lifeact-EGFP ${ }^{33}$. In wild-type neuroblasts, F-actin signals were enriched at the tip of the leading process throughout migration (Fig. 7a, left). Prior to nuclear translocation, F-actin condensation was formed ahead of the nucleus and moved towards the leading 
process, and the movement of this F-actin condensation was synchronized with centrosomal movement in wild-type neuroblasts (arrowheads in Fig. 7a, left and Supplementary Video 7). Furthermore, transient “cup-shaped” F-actin structure at the rear edge of the cell was frequently observed during nuclear translocation (a white arrow in the image at 00:09 in Fig. 7a, left and Supplementary Video 7; 17 out of 22 neuroblasts from 4 wild-type embryos; $78.7 \pm 8.7 \%$ as of the frequency of this F-actin structure in each embryo). In mDia-DKO neuroblasts, although F-actin condensation was formed ahead of the nucleus at similar intensity to wild-type neuroblasts, the movement of this F-actin condensation was impaired (arrowheads in Fig. 7a, right and Supplementary Video 8). Furthermore, F-actin accumulation at the rear edge during nuclear translocation was rarely observed in neuroblasts from mDia-DKO embryos (Fig. 7a, right and Supplementary Video 8; 2 out of 15 neuroblasts from 3 embryos; $8.33 \pm$ 8.33\% as of the frequency of this F-actin structure in each embryo). In contrast, F-actin signals at the tip of a leading process appeared undisturbed in mDia-DKO neuroblasts (Fig. 7a, right). These data suggest that mDia mediates movement of F-actin condensation towards the leading process and F-actin cup formation at the rear edge in SVZ neuroblasts.

We next examined localization of mDia by expression of EGFP-fused mDia1 or mDia3 in wild-type SVZ neuroblasts. Although overexpression of EGFP-mDia affected cell morphology and motility to some extent, neuroblasts overexpressing EGFP-mDia can still migrate with nuclear translocation. A transient enhancement of mDia1 and mDia3 signal was observed at the swelling in migrating neuroblasts (arrows in Supplementary Fig. 14). Further, both mDia1 and mDia3 accumulated at the rear edge immediately preceding nuclear translocation (arrows in Fig. 7b,c). These results suggest 
that mDia is transiently localized at the swelling and the cell rear, in which F-actin signals accumulate, in migrating SVZ neuroblasts.

\section{A role of $\mathrm{ROCK}$ in nuclear translocation during migration}

To test whether mDia cooperates with ROCK, another Rho effector regulating myosin II activity, in migration of SVZ neuroblasts, we inhibited ROCK and myosin II during migration of SVZ neuroblasts in Matrigel. Both Y-27632, a ROCK inhibitor, and blebbistatin, a myosin II inhibitor, prevented migration of SVZ neuroblasts in Matrigel (Fig. 8a,b). Time-lapse fluorescent imaging of migrating neuroblasts expressing EGFP and PACT-mKO1 was performed in the absence or presence of Y-27632 (Fig. 8c and Supplementary Video 9 and 10). With ROCK inhibition, neuroblasts showed elongated leading processes (Fig. 8c; control, $44.80 \pm 3.70 \mu \mathrm{m}, n=16$ cells; Y-27632, $62.03 \pm 6.00 \mu \mathrm{m}, n=16$ cells; $P<0.05$, $t$-test). ROCK inhibition also impaired tail retraction and induced elongation of the trailing process (Fig. 8c; control, $4.51 \pm 1.52$ $\mu \mathrm{m}, n=16$ cells; Y-27632, $22.71 \pm 7.16 \mu \mathrm{m}, n=16$ cells; $P<0.05$, $t$-test). Y-27632 spared formation of the swelling, and the maximum separation of the centrosome (thus the swelling) from the nucleus prior to nuclear translocation was not altered (Fig. 8c; control, $12.59 \pm 1.33 \mu \mathrm{m}, n=15$ cells; Y-27632, $13.15 \pm 2.32 \mu \mathrm{m}, n=9$ cells; $P>0.8$ $t$-test). However, the swelling was maintained for longer duration before the nucleus reached (control, $11.07 \pm 2.36 \mathrm{~min}, n=15$ cells; Y-27632, $35.45 \pm 4.52 \mathrm{~min}, n=11$ cells; $P<0.0001$, $t$-test), because the maximum velocity of nuclear translocation was slowed (control, $3.30 \pm 0.40 \mu \mathrm{m} \mathrm{min}^{-1}, n=18$ cells; Y-27632, $0.77 \pm 0.07 \mu \mathrm{m} \mathrm{min}^{-1}, n$ $=17$ cells; $P<0.0001, t$-test). Consequently, the maximum velocity of centrosomal 
movement preceding nuclear translocation was reduced (control, $2.53 \pm 0.28 \mu \mathrm{m} \mathrm{min}{ }^{-1}$, $n=18$ cells; Y-27632, $1.08 \pm 0.13 \mu \mathrm{m} \mathrm{min}^{-1}, n=17$ cells; $P<0.0001, t$-test). Therefore, ROCK inhibition did not affect nucleus-centrosome separation, but interfered with nuclear translocation similarly to mDia deficiency. 


\section{DISCUSSION}

Whether radial migration and tangential migration are operated by similar or distinct mechanisms within migrating neuronal precursors remains unknown. Given that mDia1 and mDia3 are expressed in mouse brains, here we generated mice lacking mDia1 and mDia3 in combination, and found that these mice are selectively impaired in tangential migration of cortical and olfactory interneuron precursors in vivo. In contrast, radial migration and consequent layer formation of cortical excitatory neurons were not impaired in mDia-DKO mice, indicating that mDia1/3 function is dispensable for radial migration. As possible cellular bases for the deficits in vivo, we found that mDia-DKO neuroblasts exhibited retarded nuclear translocation and reduced separation of the centrosome from the nucleus in vitro. Consistently, two types of mDia-dependent F-actin dynamics were observed: F-actin accumulation at the cell rear during nuclear translocation and anterograde F-actin movement towards the leading process during nucleus-centrosome separation. These data collectively suggest that mDia-mediated F-actin dynamics is critical for nuclear translocation in tangential migration through regulating movement of the centrosome and the nucleus, and that this mechanism is dispensable for radial migration in vivo (Supplementary Fig. 15). These findings provide the first example suggesting different contribution of actin dynamics to radial and tangential migration.

\section{mDia-dependent rear F-actin cup formation for migration}

The mechanism underlying nuclear translocation has been extensively studied, and the role of microtubules and dynein-mediated motor activity in nuclear translocation has been suggested. However, most of these studies were performed on radial migration, 
and the role of microtubules in tangential migration is largely unknown. Although mDia can modulate microtubule dynamics in non-neuronal cultured cells ${ }^{29-31}$, mDia-DKO neuroblasts did not show apparent abnormality in cage-like microtubule structure around the nucleus.

On the other hand, mDia-DKO neuroblasts failed to form cup-shaped F-actin structure at the rear during nuclear translocation. Since mDia is critical for nuclear translocation, this F-actin structure could generate the force pushing the nucleus from behind. Our finding that mDia is localized to the cell rear prior to nuclear translocation suggests that local supply of actin filaments by mDia is critical to form the cup-shaped F-actin structure at the rear for nuclear translocation. This mDia function seems to cooperate with ROCK, since ROCK inhibition mimicked impaired nuclear translocation as observed in mDia-DKO neuroblasts. Consistently, it was reported that myosin II and its phosphorylated (active) form are enriched at the cell rear in cortical interneuron precursors and SVZ neuroblasts undergoing nuclear translocation ${ }^{14,16,17}$, and that blebbistatin blocked nuclear translocation in these cells. These findings thus suggest that actin filaments generated by mDia are utilized to form actomyosin bundles upon ROCK activation for nuclear translocation. Given that nucleus-centrosome separation is larger in tangential migration than in radial migration, the nucleus has to move for a larger distance during nuclear translocation in tangential migration. Actomyosin-based contractility mediated by mDia and ROCK at the cell rear could promote large-distance nuclear movement in tangential migration.

\section{mDia-dependent nucleus-centrosome separation for migration}

Although nucleus-centrosome separation prior to nuclear translocation is larger in 
tangential migration than in radial migration ${ }^{3,17}$, the underlying mechanism remains unknown. We found that mDia deficiency reduced nucleus-centrosome separation before nuclear translocation. Notably, anterograde F-actin movement towards the leading process from the cell body is also impaired in mDia-DKO neuroblasts, suggesting the role of this F-actin movement in centrosomal movement prior to nuclear translocation. Given that EGFP-mDia accumulated at the swelling prior to nuclear translocation, local supply of actin filaments by mDia could be involved in this F-actin movement. Although the role of microtubules in centrosome positioning has been highlighted in non-neuronal cells, actin cytoskeleton has also been implicated in positioning the centrosome ${ }^{34}$. For example, separation and positioning of the centrosomes in spindle formation of mitotic cells are dependent on myosin $\mathrm{II}^{35}$ and diaphanous $^{36}$, a Drosophila homolog of mDia. In contrast, ROCK inhibition did not affect nucleus-centrosome separation in SVZ neuroblasts, suggesting that ROCK-mediated actomyosin contractility may not be involved, or may function redundantly with other signaling, in this process.

\section{A selective function of $\mathbf{m D i a}$ in tangential migration}

Given that both mDia and ROCK are Rho effectors, our finding about their roles in migration of SVZ neuroblasts suggests that Rho activation is required for tangential migration. In contrast, it was reported that the attenuation of Rho activity is necessary for radial migration ${ }^{37-39}$. Thus, Rho signaling appears to be differently used in radial and tangential migration. Consistently, we found that mDia deficiency does not impair, but rather promotes, radial migration. It is known that Slit-Robo signaling is critical for tangential migration ${ }^{2}$, and that this signaling activates $\mathrm{Rho}^{40}$. Previous studies showed 
that Slit is secreted from the choroid plexus, and forms a descending gradient in the direction of migration of SVZ neuroblasts ${ }^{41}$. It is thus conceivable that the cell rear of SVZ neuroblasts is exposed to Slit at a higher concentration than the cell front, leading to higher activation of Rho signaling at the cell rear. Such spatiotemporal regulation of Rho activation may provide the mechanism for the formation of cup-shaped F-actin structure by mDia at the cell rear for nuclear translocation.

In summary, our present study demonstrates that radial migration and tangential migration are operated at least in part by distinct cellular mechanisms. mDia function revealed here highlights the importance of spatiotemporal de novo F-actin formation in shaping F-actin dynamics that drives tangential migration of neuronal precursors. 


\section{ACKNOWLEDGEMENTS}

We thank Dr. Masaru Okabe for providing pCX-EGFP, Dr. Fumio Matsuzaki for providing pCAG-EGFP-C1 and pCAG-PACT-mKO1, Dr. Yuchio Yanagawa for providing GAD65/67 antisense probes, Dr. Yuichi Deguchi for providing mDia3 riboprobes, Atsushi Mizutani for animal care, Kiyoshi Tohyama for supporting genotyping and plasmid construction, and Tae Arai and Akiko Washimi for secretary help, and Kimiko Nonomura for technical assistance. R.S. thanks Dr. Akira Kakizuka for providing the opportunity to work in Kyoto University Graduate School of Medicine. R.S. was supported by the Japan Society for the Promotion of Science Research Fellowship. This work was supported in part by Grants-in-Aid for Scientific Research from the Ministry of Education, Culture, Sports, Science and Technology of Japan and a Core Research for Evolutional Science and Technology (CREST) grant from Japan Science and Technology Agency.

\section{AUTHOR CONTRIBUTIONS}

R.S., D.T., T.F. and S.N. designed the study. R.S. performed most of the experiments. H. Kamijo, R.S., H.Kiyonari, T.I. and S.N. generated mDia3-KO and mDia-DKO mice. D.T. and R.S. carried out in utero electroporation for live imaging. R.S. collected and analyzed the imaging data. N.K. and K.S. performed the whole-mount immunostaining. K.W. and H.T. provided essential advices in setting up and performing in utero electroporation, and conducted in situ hybridization. S.N. and T.F. supervised the project. R.S., T.F. and S.N. wrote the manuscript. All authors discussed the results and concurred on the contents of this manuscript. 


\section{COMPETING FINANCAL INTERESTS}

The authors declare no competing financial interests. 


\section{FIGURE LEGENDS}

Figure 1 Radial migration of excitatory cortical precursors is not impaired in mDia-DKO mice.

(a) Migration of cortical neuronal precursors pulse-labeled with EdU in wild-type mice. EdU was injected into pregnant mice carrying embryos of E15.5, and EdU-labeled cells were visualized at indicated times. The number in parenthesis indicates the time after EdU injection. Scale bar, $75 \mu \mathrm{m}$.

(b) Distribution of labeled cortical neuronal precursors in wild-type and mDia-DKO mice. EdU was injected into pregnant mice carrying embryos of E15.5, and EdU-labeled cells were visualized at E18.5. Scale bar, $75 \mu \mathrm{m}$.

(c) Quantitative analysis of distribution of EdU-labeled cells in the cerebral cortex of wild-type and mDia-DKO mice. The cerebral cortex was divided into ten equally spaced bins along the vertical axis shown in $\mathbf{b}$, and the proportion of EdU intensity in each bin was obtained and averaged across 4 sections from 2 embryos of E16.5 wild-type, 4 sections from 2 embryos of E17.5 wild-type, 21 sections form 5 embryos of E18.5 wild-type, 7 sections from 2 mice of P0 wild-type, and 9 sections from 2 embryos of E18.5 mDia-DKO. Error bars represent s.e.m.

Figure 2 Tangential migration of cortical interneurons is impaired in mDia-DKO mice.

(a) In situ hybridization of GAD65/67 in coronal sections of cerebral cortex from wild-type and mDia-DKO mice at E14.5. Dotted lines and numbers indicate equally spaced bins along the medio-lateral axis for quantification shown in $\mathbf{b}$. Arrowheads indicate the GE. Scale bars, $250 \mu \mathrm{m}$.

(b) Quantification of the number of GAD65/67-positive cells in the cerebral cortex of 
wild-type and mDia-DKO mice at E14.5 ( $n=3$ embryos for each genotype). The number of GAD65/67-positive cells in each bin shown in a was counted. ${ }^{* *} P<0.01$, *** $P<0.001$, Bonferroni's post hoc test following two-way ANOVA $\left(F_{1,20}=172.65, P\right.$ $=0.0001$ for the main genotype effect).

(c) Immunofluorescence for parvalbumin in the motor cortex from adult wild-type, mDia3-KO and mDia-DKO mice. Signals are shown in gray scale to improve the visibility. Scale bar, $250 \mu \mathrm{m}$.

(d) Quantification of the densities of parvalbumin-positive $\left(\mathrm{PV}^{+}\right)$cells in the somatosensory cortex (SSC), the motor cortex (MC) and the hippocampus (HIP) of adult wild-type, mDia3-KO and mDia-DKO mice ( $n=3-4$ mice for each genotype). Values are normalized to those of wild-type mice. ${ }^{* *} P<0.01$, *** $P<0.001$, Bonferroni's post hoc test following two-way ANOVA $\left(F_{2,20}=20.20, P=0.0001\right.$ for the main genotype effect).

Error bars represent s.e.m.

Figure 3 Migration of SVZ neuroblasts to the granule cell layer of the olfactory bulb is interrupted in mDia-DKO mice.

(a) The migratory route of SVZ neuroblasts to the olfactory bulb (red lines) shown in a sagittal brain section. The anterior (aRMS) and posterior (pRMS) levels of the RMS analyzed in $\mathbf{b}$ are shown in this diagram. CC, corpus callosum; LV, lateral ventricle.

(b) Immunofluorescence for doublecortin in coronal sections at the anterior and posterior levels indicated in a. Regions enclosed by rectangles indicate the RMS. Scale bar, $250 \mu \mathrm{m}$. 
(c) Gross appearance (top and middle panels) and Nissl-stained sagittal sections around the olfactory bulb (bottom panels) of the adult brain of mDia3-KO and mDia-DKO mice. Scale bar, $250 \mu \mathrm{m}$ only for the bottom panels.

Figure 4 mDia deficiency causes impaired migration from MGE and SVZ explants in vitro.

(a) Migration of MGE cells from explants isolated from mDia3-KO $\left(\mathrm{mDia}^{+/+} ; m \mathrm{mia}^{\text {null }}\right)$ and mDia-DKO $\left(\mathrm{mDia1}^{-/-} ; m D i a 3^{\text {null }}\right)$ mice at $48 \mathrm{~h}$ of the culture. Cells that migrated out formed bright regions surrounding the explants in phase-contrast images. Scale bar, $200 \mu \mathrm{m}$.

(b) Quantification of migration of MGE cells from the edge of MGE explants ( $n=3-8$ embryos for each genotype). The migration was quantified by measuring the area of a bright region around an explant as described in a, and is shown after normalization to the explant perimeter. ${ }^{* * *} P<0.001$, Bonferroni's post hoc test following one-way $\operatorname{ANOVA}\left(F_{4,18}=18.64, P<0.0001\right.$ for the main genotype effect $)$.

(c) Neuroblast migration from SVZ explants isolated from mDia3-KO $\left(\mathrm{mDia1}^{+/+} ; m D i a 3^{\text {null }}\right)$ and mDia-DKO $\left(\mathrm{mDia1}^{-/-} ; m D i a 3^{\text {null }}\right)$ mice at $48 \mathrm{~h}$ of the culture. Scale bar, $200 \mu \mathrm{m}$.

(d) Quantification of migration of neuroblasts from the edge of SVZ explants ( $n=3-5$ embryos for each genotype). The migration was quantified as described in $\mathbf{b}$. ${ }^{* * *} P<$ 0.001, Bonferroni's post hoc test following two-way ANOVA $\left(F_{3,11}=8.126, P=0.0039\right.$ for the main genotype effect).

(e) Time-lapse, phase-contrast imaging of neuroblast migration from SVZ explants of 
wild-type and mDia-DKO mice. Images were taken at 5 min intervals (Supplementary Video 1 (wild-type) and 2 (mDia-DKO), $n=3-4$ for each genotype), and images at every $2 \mathrm{~h}$ are shown as examples. Scale bar, $100 \mu \mathrm{m}$.

Error bars represent s.e.m.

Figure 5 Fluorescent imaging analyses of cell morphology and movement of SVZ neuroblasts.

(a) Time-lapse fluorescent imaging for migration of EGFP-labeled SVZ neuroblasts from wild-type and mDia-DKO explants. Images were acquired at 3 min intervals, and representative consecutive images at every 6 or 12 min are shown $(n=$ no less than five for each genotype). An asterisk denotes the position of an identical cell across image. Scale bar, $20 \mu \mathrm{m}$.

(b) Representative tracing of the movement of respective cell bodies. Time-lapse images for this tracing can be seen in Supplementary Video 3 (wild-type) and 4 (mDia-DKO). Scale bar, $50 \mu \mathrm{m}$.

(c) The length of a leading process of wild-type (WT) and mDia-DKO (DKO) neuroblasts. The leading process length was averaged from 10 successive images taken at 3 min intervals (see Methods).

(d) The average velocity of the movement of the cell body (soma) of wild-type (WT) and mDia-DKO (DKO) neuroblasts. Note that the motility of the cell body was decreased in mDia-DKO neuroblasts.

The number of cells is shown in each column. ${ }^{* * *} P<0.001$, $t$-test. Error bars represent s.e.m. 
Figure 6 mDia-DKO neuroblasts show reduced movement of the centrosome and the cell body.

(a) Simultaneous fluorescent imaging of the cell morphology and the centrosome of wild-type and mDia-DKO neuroblasts. Images are taken from Supplementary Video 5 (wild-type) and 6 (mDia-DKO). Signals of EGFP and PACT-mKO1, a marker for the centrosome, are shown in green and purple, respectively. White dotted lines represent the extension of the leading process. Scale bar, $20 \mu \mathrm{m}$.

(b) Temporal change in motility of the centrosome (purple) and the cell body (soma, green) in wild-type and mDia-DKO neuroblasts. Black lines at the top of each graph indicate the duration that the swelling was maintained.

Figure 7 mDia-dependent F-actin dynamics and localization of mDia in migrating SVZ neuroblasts.

(a) F-actin dynamics during migration in wild-type and mDia-DKO neuroblasts. Signals of Lifeact-EGFP and PACT-mKO1 are shown in green and purple, respectively. Images were taken from Supplementary Video 7 (wild-type) and 8 (mDia-DKO). White dotted lines indicate advancement of the rear edge of each cell. Arrowheads and arrows indicate F-actin condensation ahead of the nucleus and F-actin cup at the rear edge, respectively. Linescan profiles show the signal intensities of Lifeact-EGFP (green lines) and PACT-mKO1 (purple lines) from the rear edge to the tip of the leading process (LP) in the corresponding images. Scale bar, $20 \mu \mathrm{m}$.

(b,c) Accumulation of EGFP-mDia1 (b) and EGFP-mDia3 (c) at the cell rear in wild-type neuroblasts. mCherry or mKusabira Orange 1 (mKO1) was simultaneously 
visualized to monitor distribution of the cytoplasmic bulk. Fluorescent images were acquired at 3 min intervals indicated by the number in each frame. Linescan profiles show the signal intensities of EGFP-mDia (green lines) and mCherry or mKO1 (purple lines) in the corresponding images. Arrows indicate the accumulation of EGFP-mDia at the cell rear. Scale bar, $20 \mu \mathrm{m}$.

Figure 8 Roles of ROCK and myosin II in migration of SVZ neuroblasts.

(a) Effects of Y-27632 and blebbistatin on migration of SVZ neuroblasts in Matrigel. The images taken at $45 \mathrm{~h}$ of the culture are shown. Scale bar, $200 \mu \mathrm{m}$.

(b) Quantification of migration from the edge of SVZ explants in the absence (control) or presence of Y-27632 or blebbistatin ( $n=5-8$ explants for each treatment). ${ }^{* * *} P<$ 0.001, Bonferroni's post hoc test following two-way ANOVA $\left(F_{2,15}=35.39, P<0.0001\right.$ for the main drug effect).

(c) Time-lapse fluorescent imaging of SVZ neuroblasts treated without or with Y-27632. Images were taken from Supplementary Video 9 (control) and 10 (Y-27632). Signals of EGFP and PACT-mKO1 are shown in green and purple, respectively. Asterisks show PACT-mKO1 signals indicating the location of the centrosome. Scale bar, $20 \mu \mathrm{m}$. 


\section{METHODS}

\section{Animals}

$\mathrm{mDia1}^{-/-}$mice were generated and backcrossed to C57BL/6N more than 10 generations as previously described ${ }^{42}$. mDia3-flox mice (Acc. No. CDB0426K: http://www.cdb.riken.jp/arg/mutant\%20mice\%20list.html) and $m D i a 3^{\text {null }}$ mice were established as follows. A BAC clone of C57BL/6J containing the mDia3 gene (RP23-407E2) was purchased from BACPAC Resources Center (Oakland, CA). A 1.6-kbp fragment containing the exon 1 of mDia3 between BstEII and PstI sites was removed from this BAC clone and inserted into the PmeI site between two loxP sites of DT-A/Conditional FW vector (http://www.cdb.riken.jp/arg/cassette.html). A 6.2-kbp fragment between the PstI and BstEII sites upstream of the exon 1 was recovered from the same BAC clone, and inserted as a long arm using the AscI and NotI sites between the DT-A expression cassette and the first loxP site. A 4.7-kbp fragment between the PstI and EcoRV sites downstream of the exon 1 was recovered from the same BAC clone and inserted as a short arm using the SwaI site adjacent to the Neo expression cassette. The resultant targeting vector was linearized with the AscI site and introduced into TT2 embryonic stem cells by electroporation ${ }^{43}$. Screening of homologous recombinant ES cells and production of chimera mice are described elsewhere (http://www.cdb.riken.jp/arg/Methods.html). Chimeras were bred with C57BL/6N female mice, and resultant heterozygotes were crossed with ACTFLPe mice (Jackson Laboratory, Bar Harbor, ME) to delete Neo, and further with EIIa-Cre mice (Jackson Laboratory) to delete the exon 1 of $m D i a 3$. To eliminate the expression cassettes of Cre and FLP, $\mathrm{mDia3}^{+/-}$mice were mated with C57BL/6N mice. Resultant $\mathrm{mDia3}^{+/-}$mice lacking the Cre and FLP expression cassettes were intercrossed to produce $m D i a 3^{\text {null }}$ 
mice. $m D i a 3^{\text {null }}$ mice were backcrossed to C57BL/6N for more than 10 generations.

$\mathrm{mDia1}^{-/-} ; \mathrm{mDia3}^{\text {null }}$ (mDia-DKO) mice were generated as follows. First, $\mathrm{mDia3}^{+/-}$ females were crossed with $m$ Dia1 $1^{+/-}$males. In the offspring, $m D i a 1^{+/-} ; m D i a 3^{-/ Y}$ males were mated with $m D i a 1^{+/+} ; m D i a 3^{+/-}$females, and resultant males and females of $\mathrm{mDia1}^{+/-} ; m$ Dia3 ${ }^{\text {null }}$ genotype were intercrossed to generate $m D i a 1^{-/-} ; m D i a 3^{\text {null }}$ mice.

Genotyping analysis of mDia1-deficient allele was analyzed as previously described $^{42}$. Genotyping analysis of mDia3-deficient allele was analyzed by genomic PCR using the following primers: mDia3 F1 (5'-gcctgcaaaggaagcaatgattg-3'), mDia3 R1 (5’-ggctaggccacaagtatcagctt-3') and mDia3 R2 (5'-gatcctagtgaacctcttcgagggac-3’) (Supplementary Fig. 4).

Embryonic stages were calculated with the noon of the vaginal plug as E0.5. All animal care and use was in accordance with the National Institutes of Health Guide for the Care and Use of Laboratory Animals and was approved by the Institutional Animal Care and Use Committee of Kyoto University Graduate School of Medicine, Nagoya City University Graduate School of Medical Sciences, Kumamoto University Graduate School of Medical Science, and RIKEN Center for Developmental Biology.

\section{Plasmids}

pCX-EGFP $^{44}$ is a kind gift from Dr. Masaru Okabe (Osaka University). pCAG-EGFP-C1 and pCAG-PACT-mKO1 ${ }^{28}$ were kind gifts from Dr. Fumio Matsuzaki (RIKEN CDB). To generate pCX-mCherry, the fragment encoding EGFP in pCX-EGFP between EcoRI sites was replaced by that encoding mCherry obtained from pmCherry-C1 (Clontech, San Jose, CA) by PCR. To generate pCAG-mKO1-C1, the fragment encoding EGFP in pCAG-EGFP-C1 between AgeI and BglII sites was 
replaced by that encoding mKO1 obtained from phmKO1-MN1 (MBL, Nagoya, Japan) by PCR. To generate pCAG-Lifeact-EGFP, a synthetic oligo DNA containing the sequence encoding Lifeact ${ }^{33}$ was inserted into the NheI and AgeI sites of pCAG-EGFP-C1 in frame. To generate pCAG-EGFP-mDia1, the fragment encoding mDia1 was removed from pEGFP-mDia1 ${ }^{25}$ using BglII and SalI sites and inserted to the corresponding sites of pCAG-EGFP-C1. To generate pCAG-EGFP-mDia3, the fragment encoding mDia3 obtained from pEGFP-mDia3 ${ }^{45}$ by PCR was inserted to BspEI and BamHI sites of pCAG-EGFP-C1 in frame. All the plasmids were prepared using EndoFree Plasmid Maxi Kit (Qiagen,Valencia, CA), and the sequences were confirmed by DNA sequencing (BigDye Terminator v3.1 Cycle Sequencing Kit, Applied Biosystems, Foster City, CA).

\section{Western blotting}

Western blotting was performed as previously described ${ }^{42}$. Primary antibodies used in this study were as follows: mouse anti-mDia1 (1:1000, 610849, BD Transduction Laboratories, Franklin Lakes, NJ), rabbit anti-mDia1 ${ }^{46}$ (1:1000), rabbit anti-mDia2 ${ }^{47}$ (1:1000), goat anti-mDia3 (1:250, sc-10892, Santa Cruz Biotechnology, Santa Cruz, CA), and mouse anti-GAPDH (1:5000, AM4300, Ambion, Austin, TX).

\section{Histological analyses}

To make adult brain sections, mice were deeply anesthetized with sodium pentobarbital (Schering-Plough Animal Health, Boxmeer, Netherlands) at $50 \mathrm{mg} / \mathrm{kg}$ intraperitoneally (i.p.), and were transcardially perfused with $4 \%$ paraformaldehyde in 0.1 M sodium phosphate buffer ( $\mathrm{PB}, \mathrm{pH}$ 7.4). Brains were removed and post-fixed in 
the same fixative for overnight. For embryonic and neonatal brain sections, fresh brains were rapidly removed and immersed in the above fixative for overnight. The brains were cryoprotected by $0.1 \mathrm{M}$ PB containing $30 \%$ sucrose, and were frozen in Tissue-Tek OCT compounds (Sakura, Flemingweg, Netherlands). Sagittal or coronal slices of 30- $\mu$ m thickness were then made using the cryostat (HM500 OM, Carl Zeiss, Jena, Germany).

Nissl staining was performed using standard procedures with $0.1 \%$ Cresyl Violet solution (Muto Pure Chemicals, Tokyo, Japan). For immunostaining, brain sections were incubated in blocking buffer (3-10\% normal donkey serum and 0.3\% Triton X-100 in PBS) for $1 \mathrm{~h}$ at RT, and then in blocking buffer containing primary antibodies overnight at $4^{\circ} \mathrm{C}$. After several washes in PBS containing $0.3 \%$ Triton X-100, the sections were incubated with blocking buffer containing Alexa Fluor 488-conjugated or Alexa Fluor 594-conjugated secondary antibodies (1:200, Invitrogen, Carlsbad, CA) for $2 \mathrm{~h}$ at RT. Whole-mount staining of the lateral wall of the lateral ventricles was performed as previously described ${ }^{41}$. The following primary antibodies were used: rabbit anti-DIAPH2 (i.e. mDia3; 1:200, HPA005647, Sigma, St. Louis, MO), goat anti-doublecortin (1:200, sc-8066, Santa Cruz), rabbit anti-CDP antibody (i.e., Cux1; 1:200, sc-13024, Santa Cruz), rat anti-Ctip2 (1:500, ab18465, Abcam, Cambridge, UK), rabbit anti-Foxp2 (1:1000, ab16046, Abcam), rabbit anti-GABA (1:3000, A 2052, Sigma), rabbit anti-Parvalbumin (1:2000, ab11427, Abcam) and mouse anti-Reelin (1:500, MAB5364, Millipore). Fluorescent images were acquired using a TCS SP5 confocal microscope (Leica Microsystems, Wetzlar, Germany). For cell counting in Fig. 2d and Supplementary Fig. 6c,d, images were binarized and counted using MetaMorph (Universal Imaging, Buckinghamshire, UK). 
In situ hybridization was performed as previously described ${ }^{48}$. To generate mDia1 riboprobes, a 880-bp fragment containing a portion of mDia1 cDNA (81-959 nucleotide; Accession No. NM_007858) was inserted into between XhoI and NotI sites of pBluescript-SK(-) in both orientations. To generate mDia3 riboprobes, a 1031-bp fragment of mDia3 cDNA (200-1230 nucleotide; Accession No. NM_017398) was inserted into pCR4-TOPO (Invitrogen). GAD65 and GAD67 antisense probes were kind gifts from Dr. Yuchio Yanagawa (Gunma University). Images were acquired using a BX-50 upright microscope (Olympus, Tokyo, Japan) equipped with a color CCD camera (Optronics, Tokyo, Japan).

\section{EdU pulse-labelling and detection of EdU-incorporating cells}

5-ethynyl-2'-deoxyuridine (EdU, Invitrogen) was dissolved in PBS. Pregnant mice carrying embryos of E13.5 or E15.5 and adult mice were injected i.p. with EdU (15 $\mathrm{mg} / \mathrm{kg}$ ) for labeling. For the analysis of radial migration in Fig. 1, brains were removed and fixed at the indicated age. For the analysis of cell proliferation in Supplementary Fig. 11, brains were removed and fixed one hour after injection. The fixed brains were processed to cryosection as described above. EdU detection was performed using a Click-iT EdU Imaging kit (Invitrogen) according to the manufacturer's protocol with minor modification.

\section{Explant culture}

MGE and SVZ explants were prepared from E14.5 and E18-19, respectively. mDia-DKO embryos were taken from mating between $\mathrm{mDia1}^{+/} ; \mathrm{mDia}^{\text {null }}$ pairs. Explants from different embryos were separately prepared, and only those of 
appropriate genotypes were chosen for further experiments according to genotypes determined by genomic PCR of each embryo. Explants were prepared and cultured as described previously ${ }^{12}$. Briefly, brains taken from embryos were embedded in $4 \%$ low-melting agarose gel, and sliced using vibratome (DTK-3000W, Dosaka EM, Kyoto, Japan) at $300-\mu \mathrm{m}$ thickness. MGE or SVZ, located lateral to the lateral ventricle, was dissected and cut into small pieces under the stereomicroscope. These pieces were embedded on glass-bottom dishes (Matsunami Glass, Osaka, Japan) in 75\% Matrigel (a 3:1 mixture of Matrigel (BD Bioscience, San Jose, CA) and L-15 medium (Invitrogen)). The resultant explants were cultured in Neurobasal medium (Invitrogen) supplemented with 10\% fetal bovine serum (PAA Laboratories, Pasching, Austria), 2 mM L-glutamine, 2\% B-27 (Invitrogen), and penicillin-streptomycin mixture (Nacalai, Kyoto, Japan), at $37^{\circ} \mathrm{C}$ in a humidified air containing $5 \% \mathrm{CO}_{2}$.

For experiments in Fig. 8, drugs were added to the medium at the beginning of the culture and used at the following concentrations: Y-27632 (30 $\mu \mathrm{M}$, Calbiochem, San Diego, CA) and blebbistatin (100 $\mu \mathrm{M}$, Tocris, Ballwin, MO).

\section{Phase-contrast and fluorescent time-lapse imaging}

For experiments in Fig. 4 and Fig. 8, phase-contrast images were acquired using an Axiovert 25-inverted microscope (Carl Zeiss) equipped with a digital camera (Olympus). For experiments in Fig. 4e and Supplementary Video 1 and 2, a time series of phase-contrast images of SVZ explants at 5-min intervals was acquired through a 20×, 0.40 N.A. objective lens attached to an inverted microscope (Leica Application Solution Multi-Dimensional Workstation, Leica Microsystems). 
For experiments in Fig. 5-8 and Supplementary Video 3-10, time-lapse fluorescent confocal images were obtained at 3-min intervals for 30-120 min through a 40×, 1.25 N.A. Plan-Apochromat objective lens attached with a TCS SP5 confocal microscope (Leica Microsystems). At every time point, confocal images at multiple consecutive optical Z planes (6-15 levels) at 1.5- $\mu \mathrm{m}$ step intervals were collected and projected to the horizontal plane for subsequent analyses. During image acquisition, SVZ explants were maintained at $37^{\circ} \mathrm{C}$ and $5 \% \mathrm{CO}_{2}$ in a humidified chamber (Tokken, Kashiwa, Chiba, Japan) attached with an inverted microscope.

Images were analyzed and quantified with ImageJ software (National Institute of Health, Bethesda, MD) and MetaMorph (Universal Imaging, Buckinghamshire, UK).

\section{In utero electroporation}

In utero electroporation for fluorescent imaging of SVZ neuroblasts in vitro was performed as described previously ${ }^{49}$ with modifications. Briefly, plasmid DNA colored by Fast Green $(0.1 \%)$ was injected by pressure into the right lateral ventricle of an embryo through a glass pipette connected to the Pneumatic PicoPump (World Precision Instruments, Sarasota, FL). Electroporation (5 pulses of $33 \mathrm{mV}, 50 \mathrm{~ms}$ ) was then applied using an electroporator (CUY21, BEX, Tokyo, Japan). To introduce plasmids into SVZ efficiently, we optimized the angle of an electrode. The concentrations of plasmids used for electroporation were as follows: $1.0 \mu \mathrm{g} / \mu \mathrm{l}$ (pCX-EGFP, pCX-mCherry, pCAG-mKO1-C1, pCAG-PACT-mKO1, and pCAG-Lifeact-EGFP), and $1.5 \mu \mathrm{g} / \mu \mathrm{l}$ (pCAG-EGFP-mDia1 and pCAG-EGFP-mDia3). At $24 \mathrm{~h}$ after electroporation, SVZ was dissected for explant culture. 


\section{Quantification of processes and branches in SVZ neuroblasts}

For quantification of processes (Fig. 5c and Supplementary Fig. 12), migration of SVZ neuroblasts expressing EGFP and PACT-mKO1 was monitored by fluorescent time-lapse imaging taken at 3-min interval for $30 \mathrm{~min}$. A process to which the centrosome is directed is defined as the leading process, and a process from the opposite site as the trailing process. Processes that bifurcate from the leading process are defined as side branches. The number of a process or branch that is present in all 10 consecutive frames is determined as 1 , and that is present only in one frame as 0.1 . The length of a process is an average value of those in the 10 frames.

\section{Immunostaining in two-dimensional culture of SVZ neuroblasts}

SVZ was dissected from E18-19 embryo as described above, and digested with trypsin-EDTA (Invitrogen). After trituration, the dissociated cells were plated on glass-bottom dishes coated with 75\% Matrigel and cultured in the explant culture medium described above at $37^{\circ} \mathrm{C}$ in a humidified air containing $5 \% \quad \mathrm{CO}_{2}$. Immunofluorescence for dissociated cultured cells was performed as described previously $^{50}$. The following primary antibodies were used: mouse anti- $\alpha$-tubulin (1:1000, clone DM1A, Sigma), mouse anti-acetylated tubulin (1:1000, clone 6-11B-1, Sigma), and rat anti-tyrosinated tubulin (1:1000, clone YL1/2, Abcam). Alexa Fluor 488-conjugated anti-mouse and Alexa Fluor 594-conjugated anti-rat IgG antibodies (1:200, Invitrogen) were used as secondary antibodies. Fluorescent images were acquired using a TCS SP5 confocal microscope (Leica Microsystems).

\section{Statistical analyses}


All data are shown as means \pm s.e.m. Comparison of two groups was analyzed using unpaired two-tailed Student's t-test. For comparison of more than two groups, one-way or two-way ANOVA was performed and followed by Bonferroni's post hoc test for evaluation of pairwise group differences. A value of $P<0.05$ was considered statistically significant. The analyses were performed by Prism 4.0 software (GraphPad, San Diego, CA). 


\section{REFFERENCES}

1. Ghashghaei, H.T., Lai, C. \& Anton, E.S. Neuronal migration in the adult brain: are we there yet? Nat. Rev. Neurosci. 8, 141-151 (2007).

2. Marin, O. \& Rubenstein, J.L. Cell migration in the forebrain. Annu. Rev. Neurosci. 26, 441-483 (2003).

3. Metin, C., Vallee, R.B., Rakic, P. \& Bhide, P.G. Modes and mishaps of neuronal migration in the mammalian brain. J. Neurosci. 28, 11746-11752 (2008).

4. Alvarez-Buylla, A. \& Garcia-Verdugo, J.M. Neurogenesis in adult subventricular zone. J. Neurosci. 22, 629-634 (2002).

5. Lledo, P.M., Merkle, F.T. \& Alvarez-Buylla, A. Origin and function of olfactory bulb interneuron diversity. Trends Neurosci. 31, 392-400 (2008).

6. Kawauchi, T. \& Hoshino, M. Molecular pathways regulating cytoskeletal organization and morphological changes in migrating neurons. Dev. Neurosci. 30, 36-46 (2008).

7. Marin, O., Valdeolmillos, M. \& Moya, F. Neurons in motion: same principles for different shapes? Trends Neurosci. 29, 655-661 (2006).

8. Xie, Z., Sanada, K., Samuels, B.A., Shih, H. \& Tsai, L.H. Serine 732 phosphorylation of FAK by Cdk5 is important for microtubule organization, nuclear movement, and neuronal migration. Cell 114, 469-482 (2003).

9. Solecki, D.J., Model, L., Gaetz, J., Kapoor, T.M. \& Hatten, M.E. Par6 $\alpha$ signaling controls glial-guided neuronal migration. Nat. Neurosci. 7, 1195-1203 (2004).

10. Tsai, J.W., Bremner, K.H. \& Vallee, R.B. Dual subcellular roles for LIS1 and dynein in radial neuronal migration in live brain tissue. Nat. Neurosci. 10, 970-979 (2007).

11. O'Rourke, N.A., Dailey, M.E., Smith, S.J. \& McConnell, S.K. Diverse migratory pathways in the developing cerebral cortex. Science 258, 299-302 (1992).

12. Wichterle, H., Garcia-Verdugo, J.M. \& Alvarez-Buylla, A. Direct evidence for homotypic, glia-independent neuronal migration. Neuron 18, 779-791 (1997).

13. Marin, O. \& Rubenstein, J.L. A long, remarkable journey: tangential migration in the telencephalon. Nat. Rev. Neurosci. 2, 780-790 (2001).

14. Schaar, B.T. \& McConnell, S.K. Cytoskeletal coordination during neuronal migration. Proc. Natl. Acad. Sci. USA 102, 13652-13657 (2005).

15. Baudoin, J.P., Alvarez, C., Gaspar, P. \& Metin, C. Nocodazole-induced changes in microtubule dynamics impair the morphology and directionality of migrating medial ganglionic eminence cells. Dev. Neurosci. 30, 132-143 (2008). 
16. Martini, F.J. \& Valdeolmillos, M. Actomyosin contraction at the cell rear drives nuclear translocation in migrating cortical interneurons. J. Neurosci. 30, 8660-8670 (2010).

17. Bellion, A., Baudoin, J.P., Alvarez, C., Bornens, M. \& Metin, C. Nucleokinesis in tangentially migrating neurons comprises two alternating phases: forward migration of the Golgi/centrosome associated with centrosome splitting and myosin contraction at the rear. J. Neurosci. 25, 5691-5699 (2005).

18. Hall, A. Rho GTPases and the actin cytoskeleton. Science 279, 509-514 (1998).

19. Govek, E.E., Newey, S.E. \& Van Aelst, L. The role of the Rho GTPases in neuronal development. Genes Dev.19, 1-49 (2005).

20. Nguyen, L., Besson, A., Roberts, J.M. \& Guillemot, F. Coupling cell cycle exit, neuronal differentiation and migration in cortical neurogenesis. Cell Cycle 5, 2314-2318 (2006).

21. Govek, E.E., Hatten, M.E. \& Van Aelst, L. The role of Rho GTPase proteins in CNS neuronal migration. Dev. Neurobiol. 71, 528-553 (2011).

22. Narumiya, S., Ishizaki, T. \& Watanabe, N. Rho effectors and reorganization of actin cytoskeleton. FEBS Lett. 410, 68-72 (1997).

23. Kimura, K., et al. Regulation of myosin phosphatase by Rho and Rho-associated kinase (Rho-kinase). Science 273, 245-248 (1996).

24. Ishizaki, T., et al. p160ROCK, a Rho-associated coiled-coil forming protein kinase, works downstream of Rho and induces focal adhesions. FEBS Lett. 404, 118-124 (1997).

25. Watanabe, N., Kato, T., Fujita, A., Ishizaki, T. \& Narumiya, S. Cooperation between mDia1 and ROCK in Rho-induced actin reorganization. Nat. Cell Biol. 1, 136-143 (1999).

26. Higashida, C., et al. Actin polymerization-driven molecular movement of mDia1 in living cells. Science 303, 2007-2010 (2004).

27. Campellone, K.G. \& Welch, M.D. A nucleator arms race: cellular control of actin assembly. Nat. Rev. Mol. Cell Biol. 11, 237-251 (2010).

28. Konno, D., et al. Neuroepithelial progenitors undergo LGN-dependent planar divisions to maintain self-renewability during mammalian neurogenesis. Nat. Cell Biol. 10, 93-101 (2008).

29. Wen, Y., et al. EB1 and APC bind to mDia to stabilize microtubules downstream of Rho and promote cell migration. Nat. Cell Biol. 6, 820-830 (2004).

30. Ishizaki, T., et al. Coordination of microtubules and the actin cytoskeleton by the Rho effector mDia1. Nat. Cell Biol. 3, 8-14 (2001). 
31. Palazzo, A.F., Cook, T.A., Alberts, A.S. \& Gundersen, G.G. mDia mediates Rho-regulated formation and orientation of stable microtubules. Nat. Cell Biol. 3, 723-729 (2001).

32. Westermann, S. \& Weber, K. Post-translational modifications regulate microtubule function. Nat. Rev. Mol. Cell Biol. 4, 938-947 (2003).

33. Riedl, J., et al. Lifeact: a versatile marker to visualize F-actin. Nat. Methods 5, 605-607 (2008).

34. Vaughan, S. \& Dawe, H.R. Common themes in centriole and centrosome movements. Trends Cell Biol. 21, 57-66 (2011).

35. Rosenblatt, J., Cramer, L.P., Baum, B. \& McGee, K.M. Myosin II-dependent cortical movement is required for centrosome separation and positioning during mitotic spindle assembly. Cell 117, 361-372 (2004).

36. Cao, J., Crest, J., Fasulo, B. \& Sullivan, W. Cortical Actin Dynamics Facilitate Early-Stage Centrosome Separation. Curr. Biol. (2010).

37. Nguyen, L., et al. p27kip1 independently promotes neuronal differentiation and migration in the cerebral cortex. Genes Dev. 20, 1511-1524 (2006).

38. Ge, W., et al. Coupling of cell migration with neurogenesis by proneural bHLH factors. Proc. Natl. Acad. Sci. USA 103, 1319-1324 (2006).

39. Pacary, E., et al. Proneural Transcription Factors Regulate Different Steps of Cortical Neuron Migration through Rnd-Mediated Inhibition of RhoA Signaling. Neuron 69, 1069-1084 (2011).

40. Patel, B.N. \& Van Vactor, D.L. Axon guidance: the cytoplasmic tail. Curr. Opin. Cell Biol. 14, 221-229 (2002).

41. Sawamoto, K., et al. New neurons follow the flow of cerebrospinal fluid in the adult brain. Science 311, 629-632 (2006).

42. Sakata, D., et al. Impaired T lymphocyte trafficking in mice deficient in an actin-nucleating protein, mDia1. J. Exp. Med. 204, 2031-2038 (2007).

43. Yagi, T., et al. A novel ES cell line, TT2, with high germline-differentiating potency. Anal. Biochem. 214, 70-76 (1993).

44. Niwa, H., Yamamura, K. \& Miyazaki, J. Efficient selection for high-expression transfectants with a novel eukaryotic vector. Gene 108, 193-199 (1991).

45. Yasuda, S., et al. Cdc42 and mDia3 regulate microtubule attachment to kinetochores. Nature 428, 767-771 (2004).

46. Watanabe, N., et al. p140mDia, a mammalian homolog of Drosophila diaphanous, is a target protein for Rho small GTPase and is a ligand for profilin. EMBO J. 16, 3044-3056 (1997). 
47. Watanabe, S., et al. mDia2 induces the actin scaffold for the contractile ring and stabilizes its position during cytokinesis in NIH 3T3 cells. Mol. Biol. Cell 19, 2328-2338 (2008).

48. Masahira, N., et al. Olig2-positive progenitors in the embryonic spinal cord give rise not only to motoneurons and oligodendrocytes, but also to a subset of astrocytes and ependymal cells. Dev. Biol. 293, 358-369 (2006).

49. Saito, T. \& Nakatsuji, N. Efficient gene transfer into the embryonic mouse brain using in vivo electroporation. Dev. Biol. 240, 237-246 (2001).

50. Umeshima, H., Hirano, T. \& Kengaku, M. Microtubule-based nuclear movement occurs independently of centrosome positioning in migrating neurons. Proc. Natl. Acad. Sci. USA 104, 16182-16187 (2007). 
a

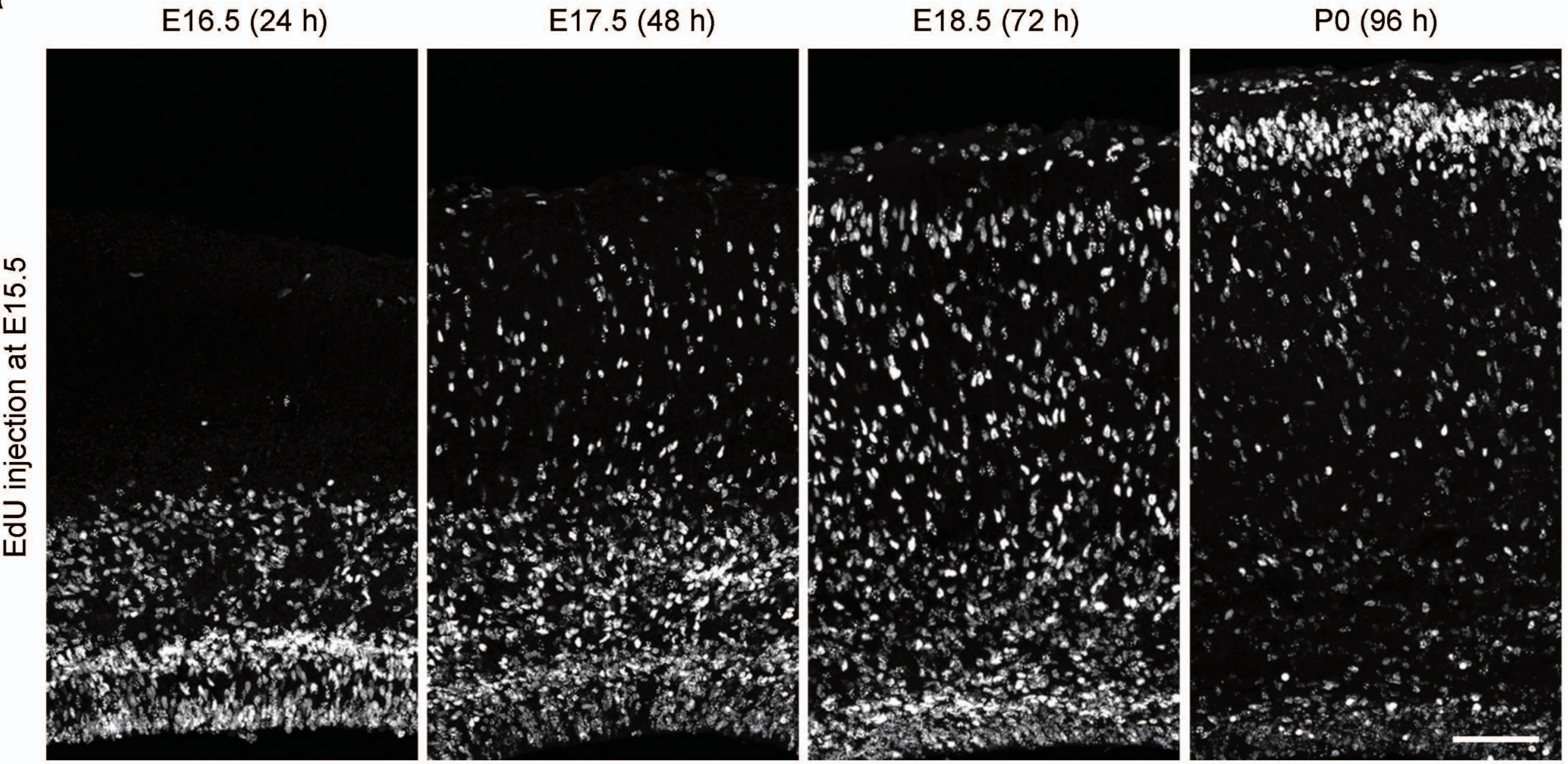

b

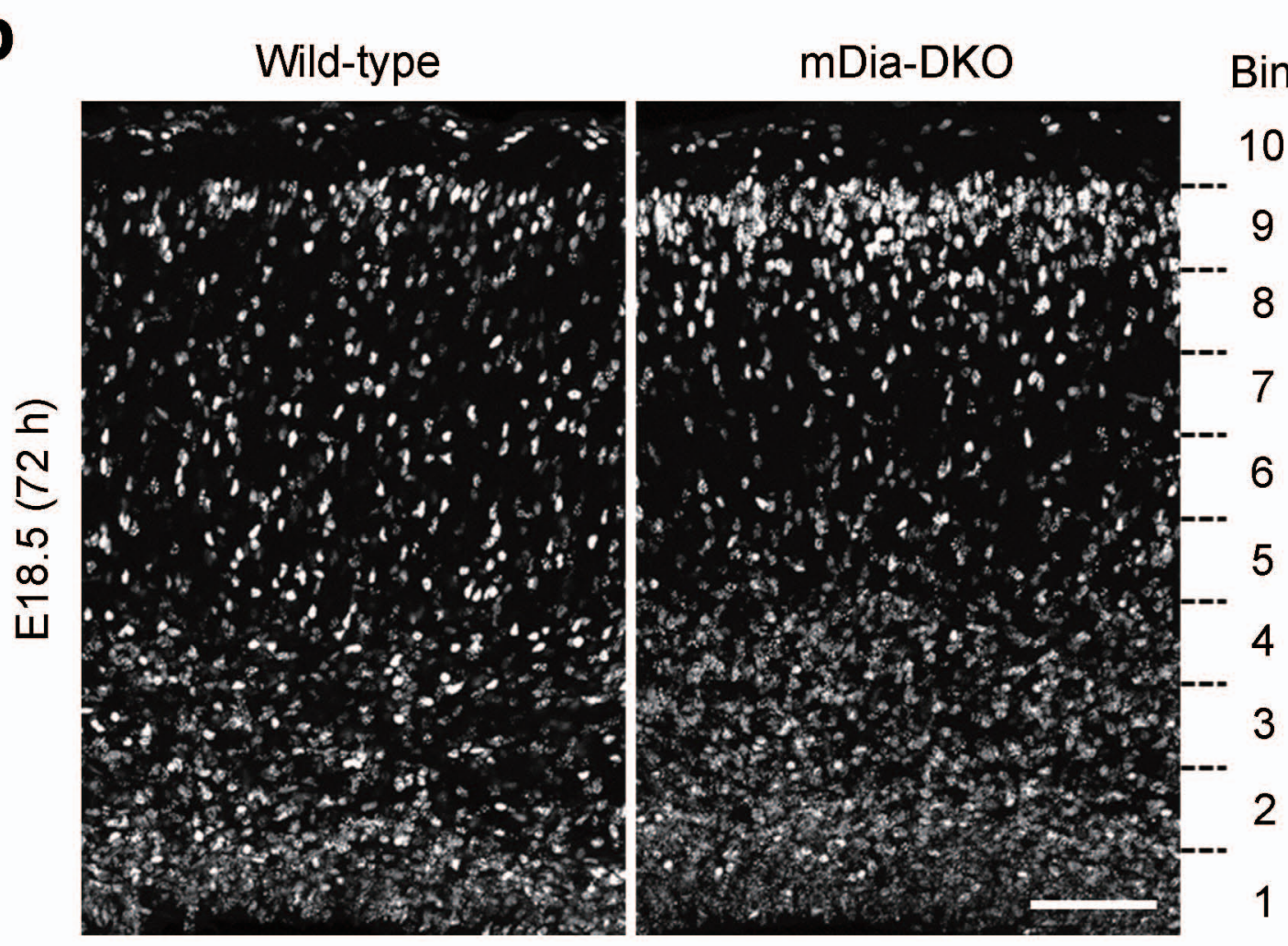

C

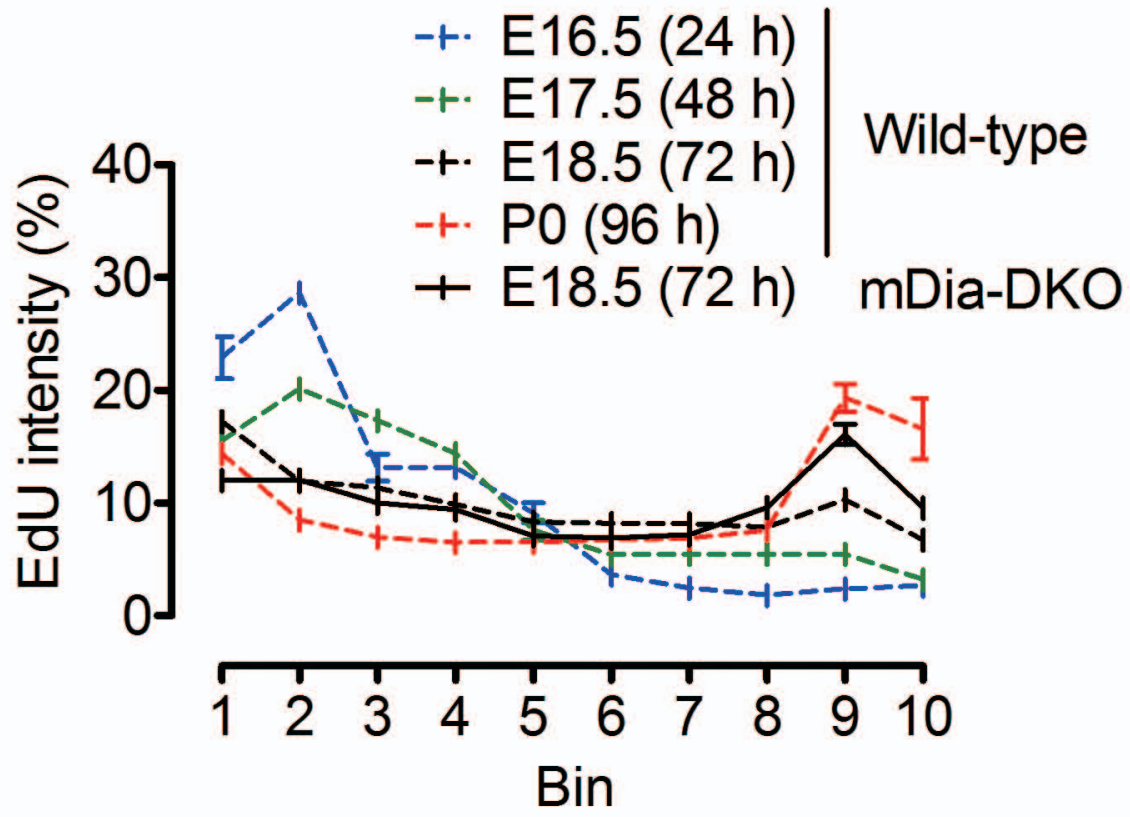

Figure 1 


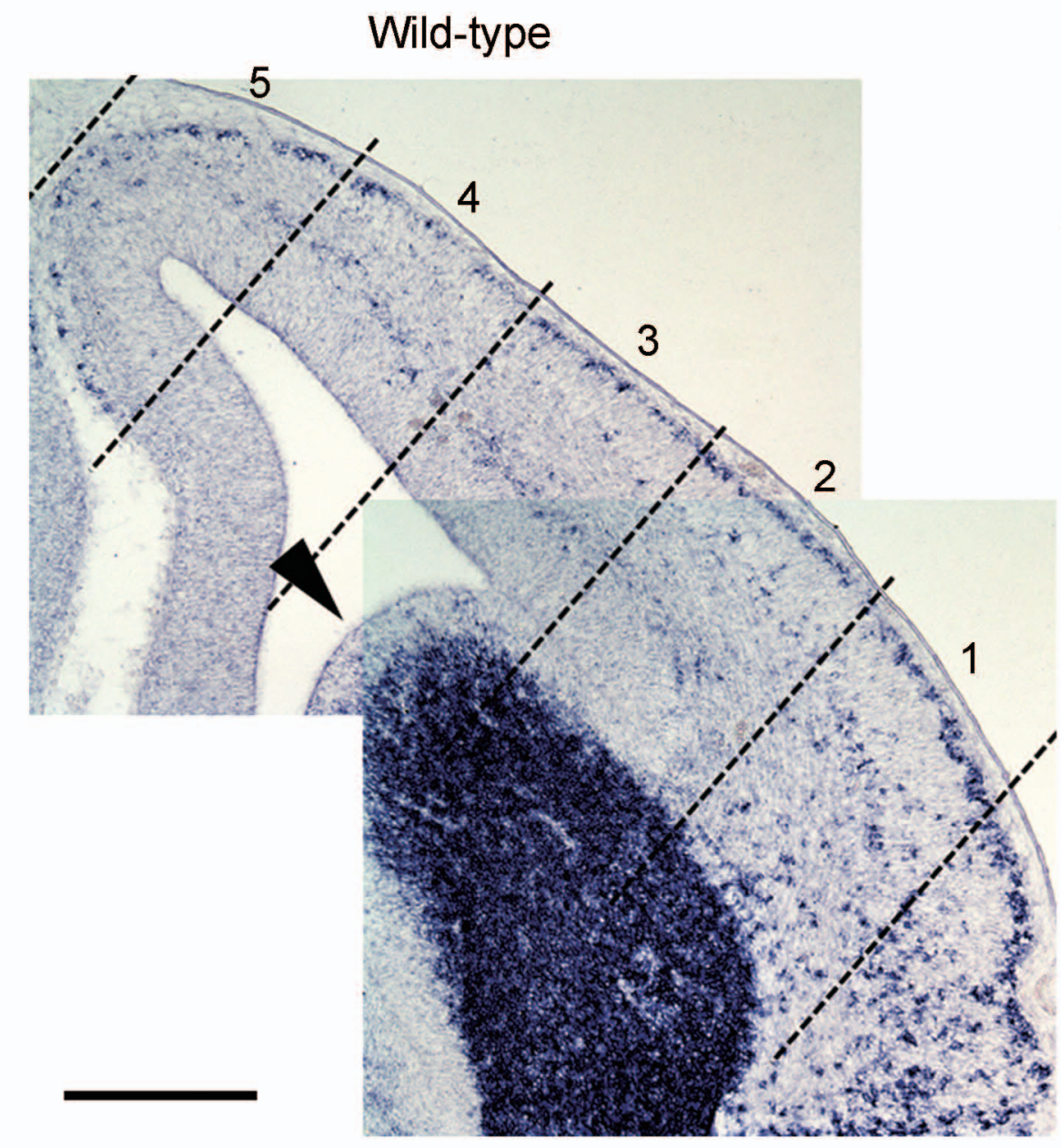

C

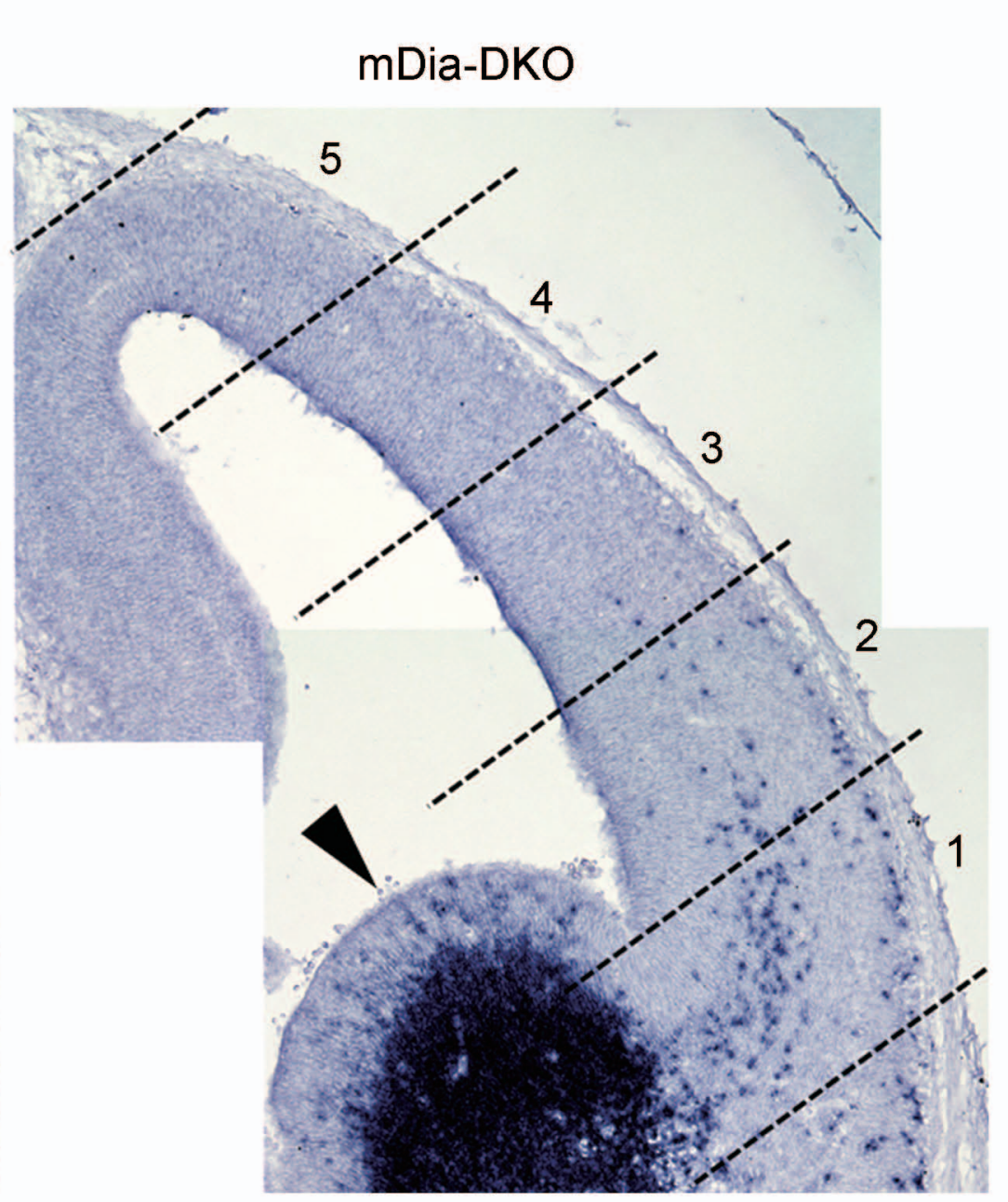

b

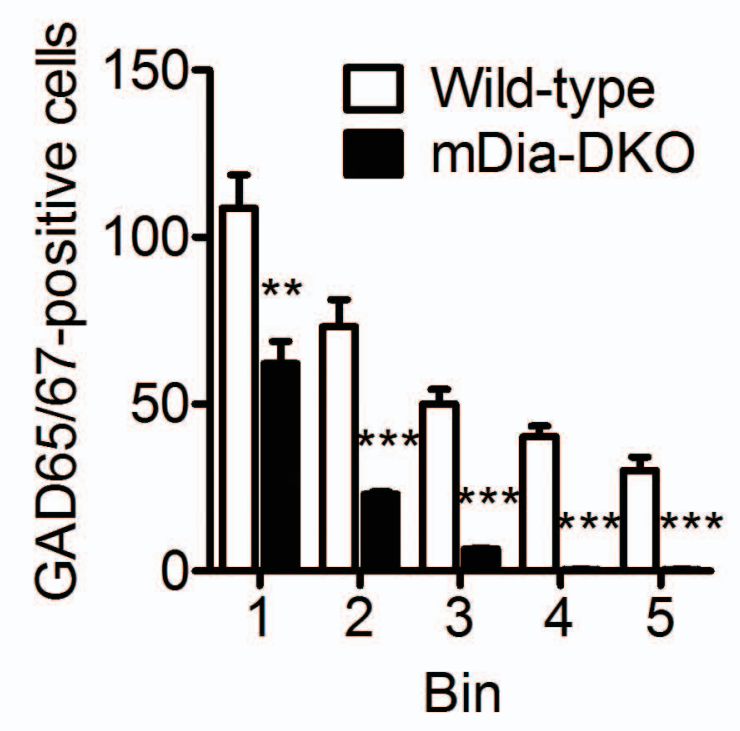

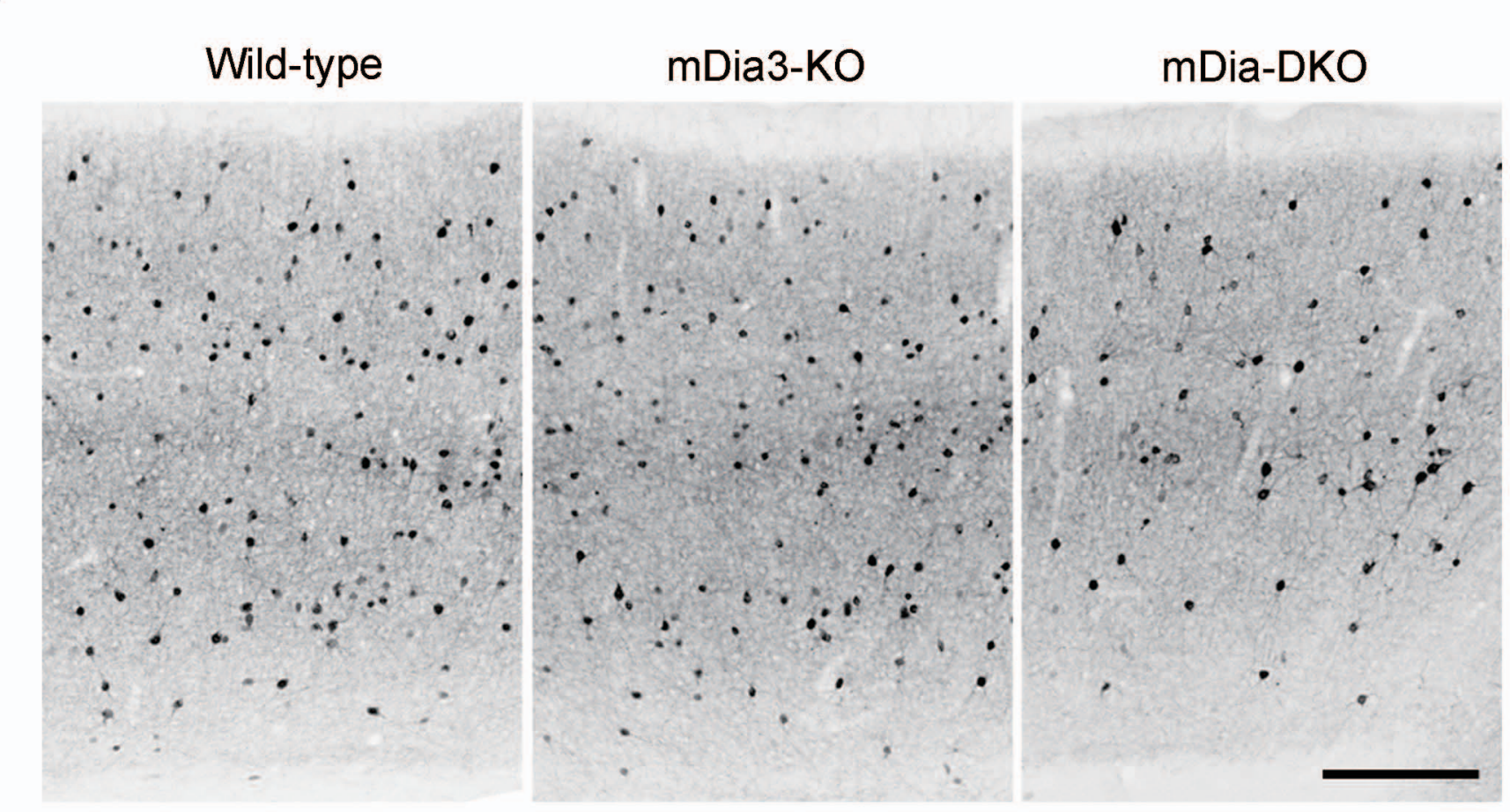

d

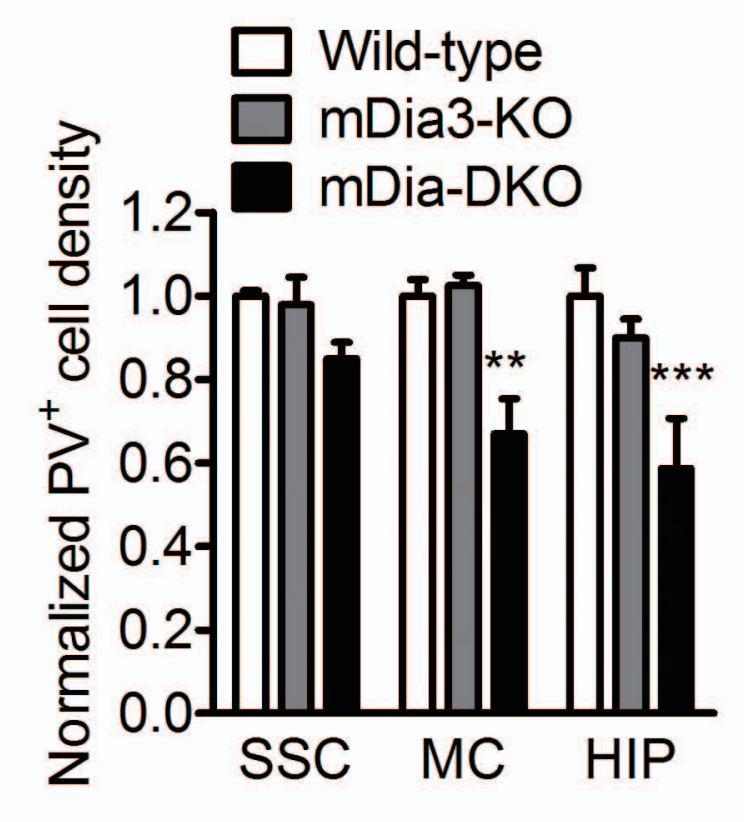

Figure 2 
a

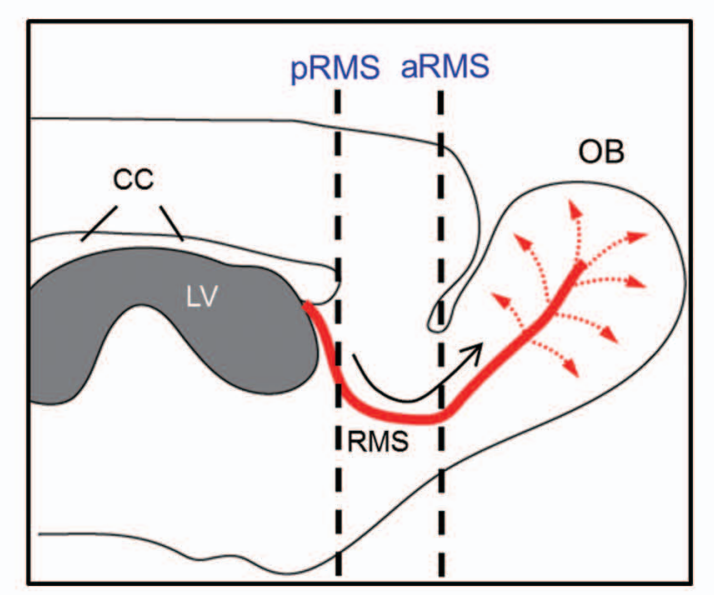

b

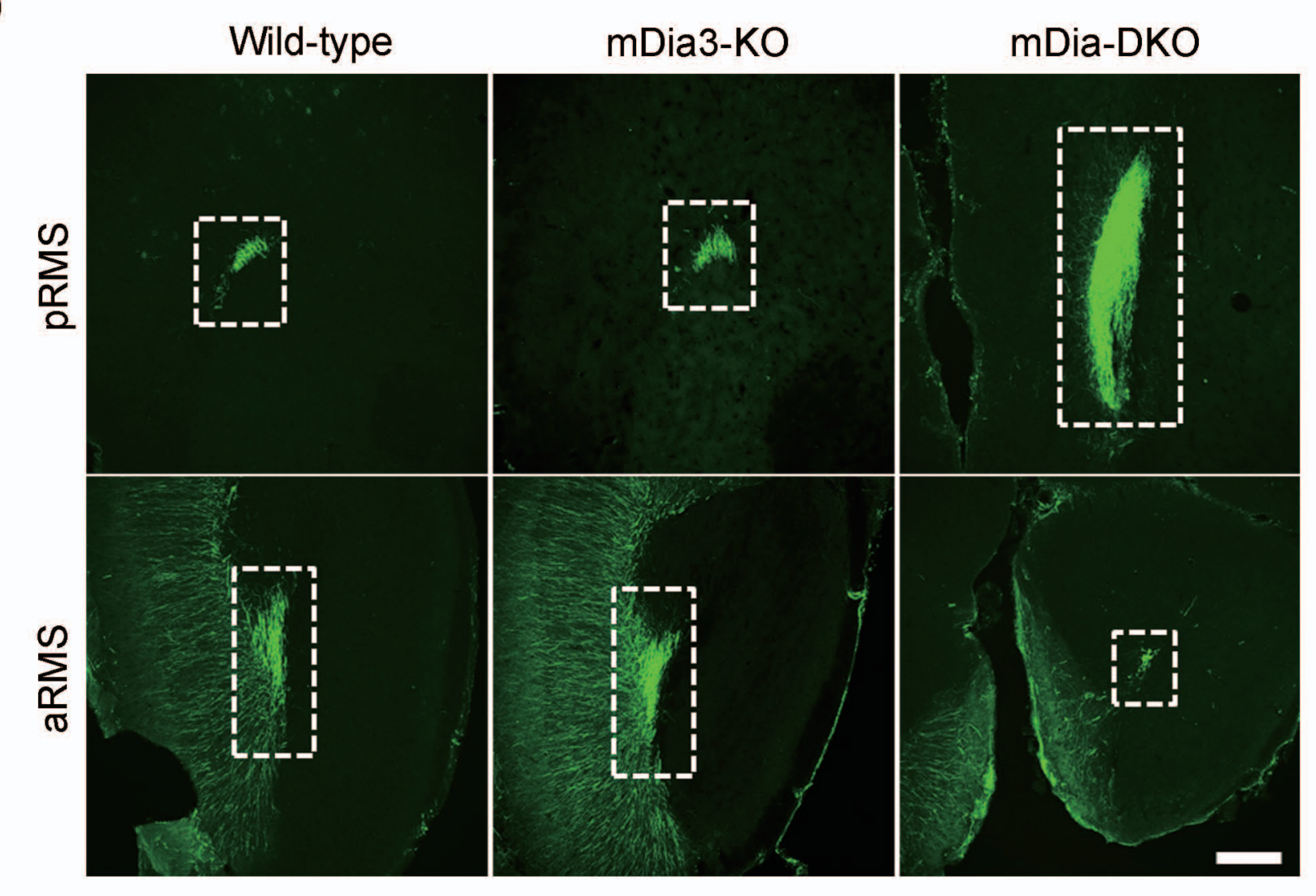

C

mDia3-KO
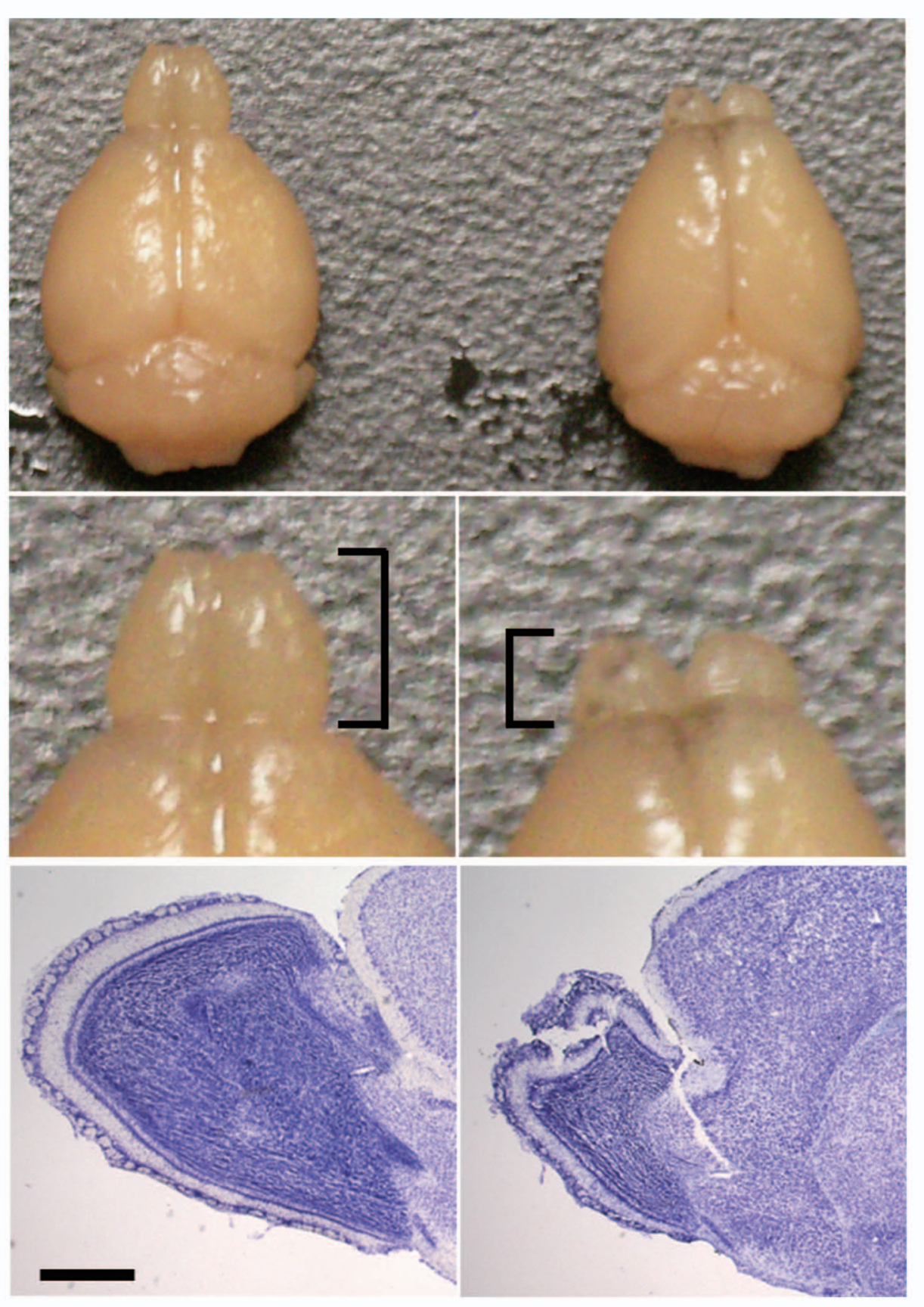

Figure 3 
a

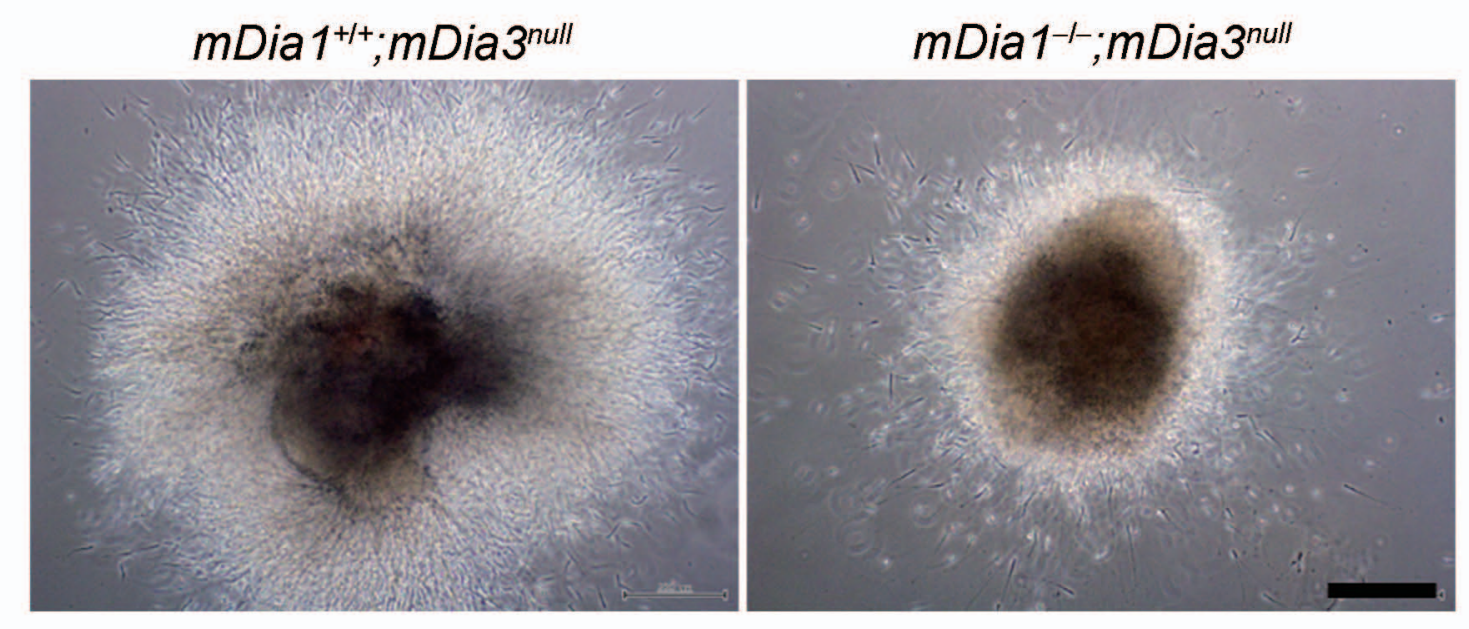

C

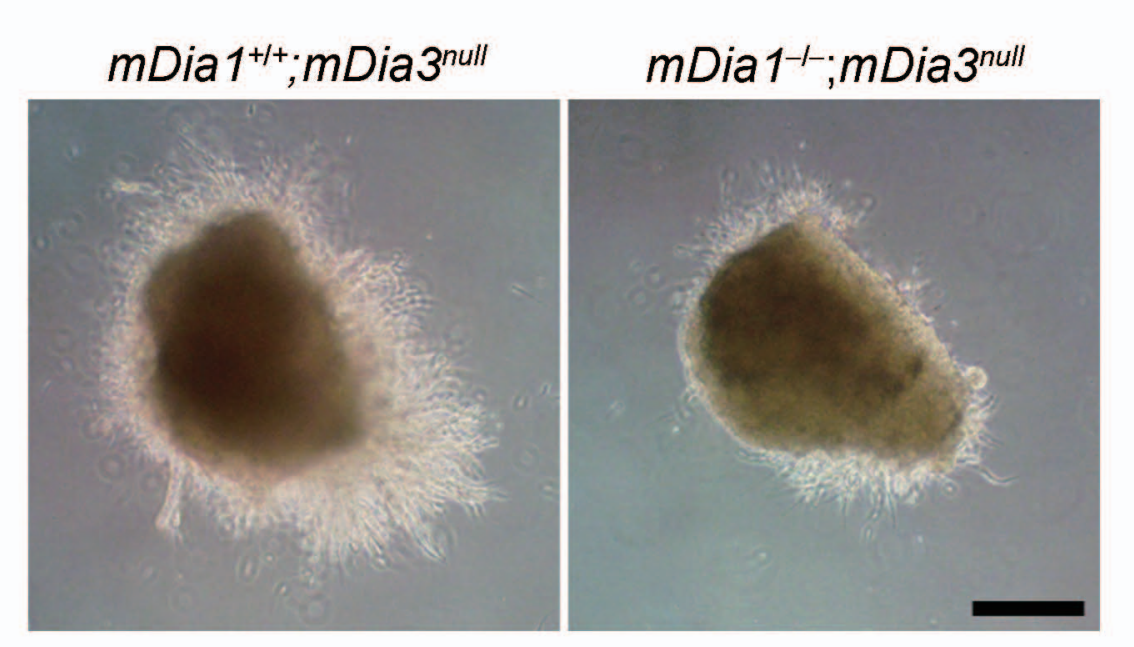

e

\section{Wild-type}
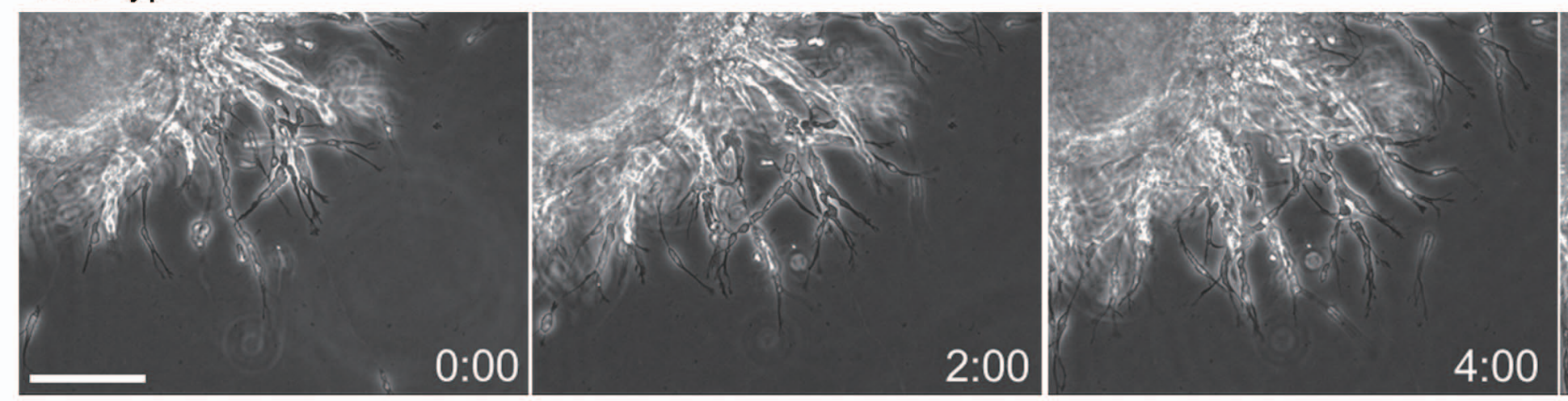

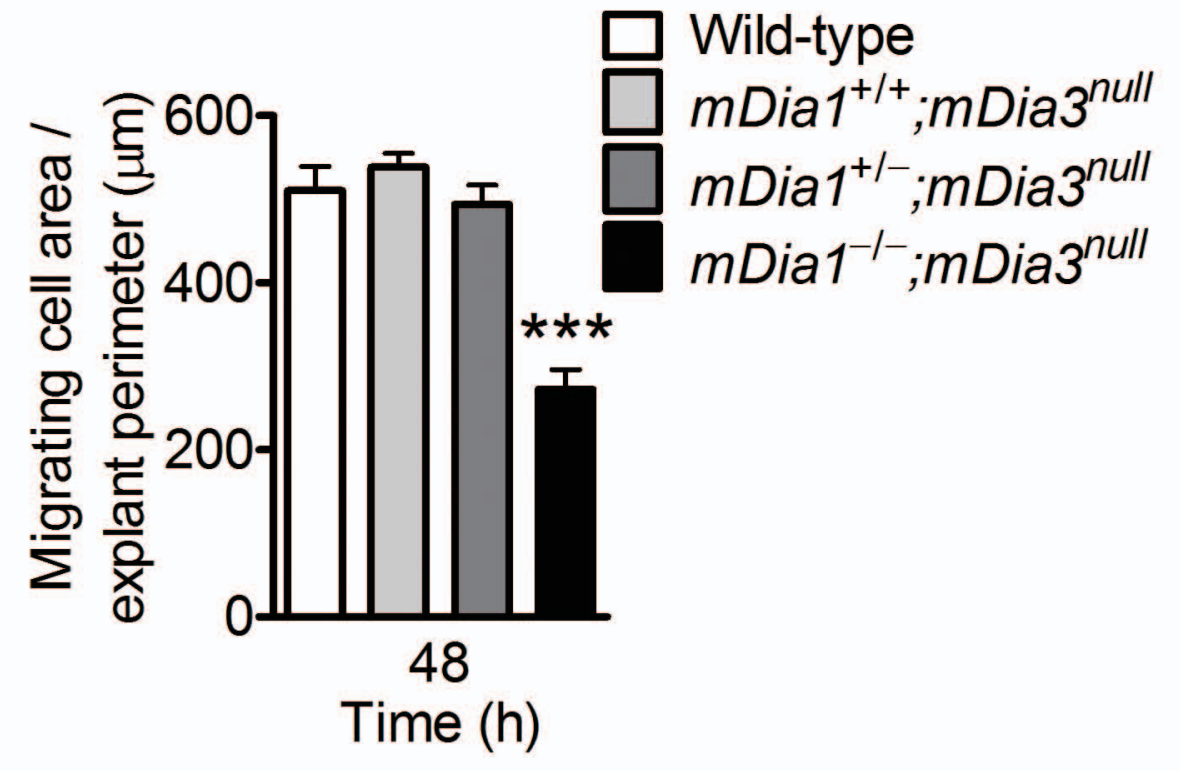

d

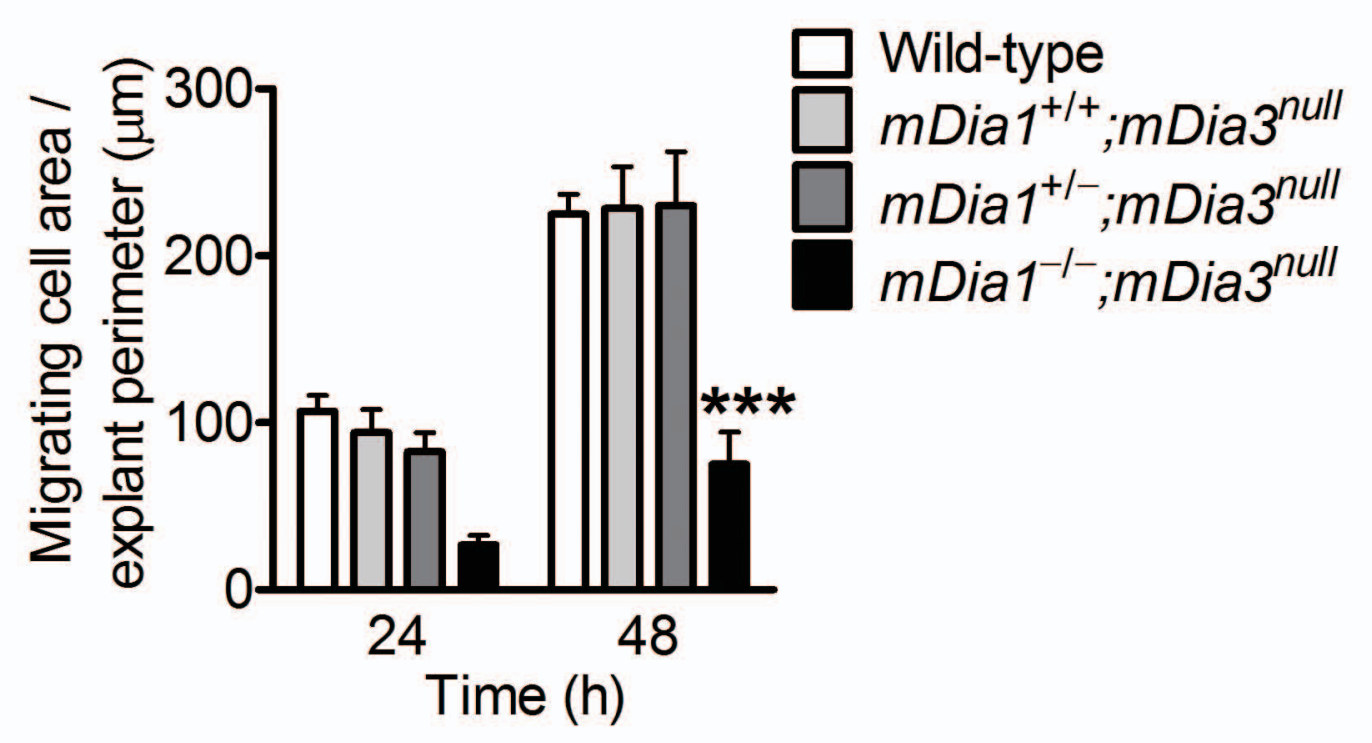

mDia-DKO
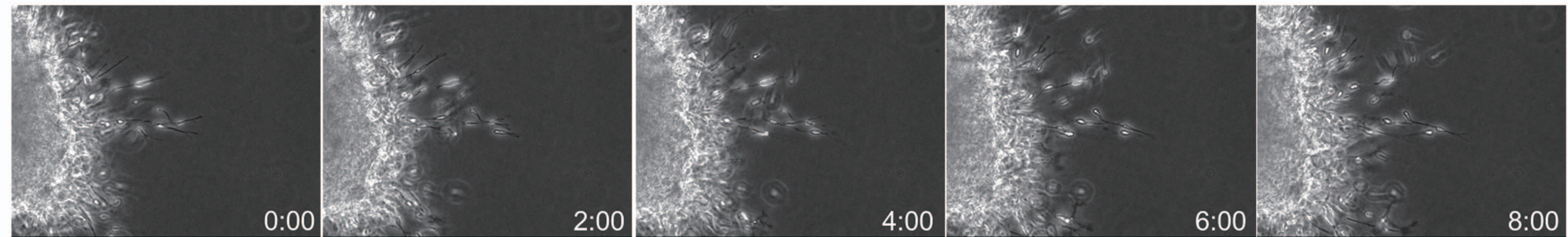

Figure 4 
a
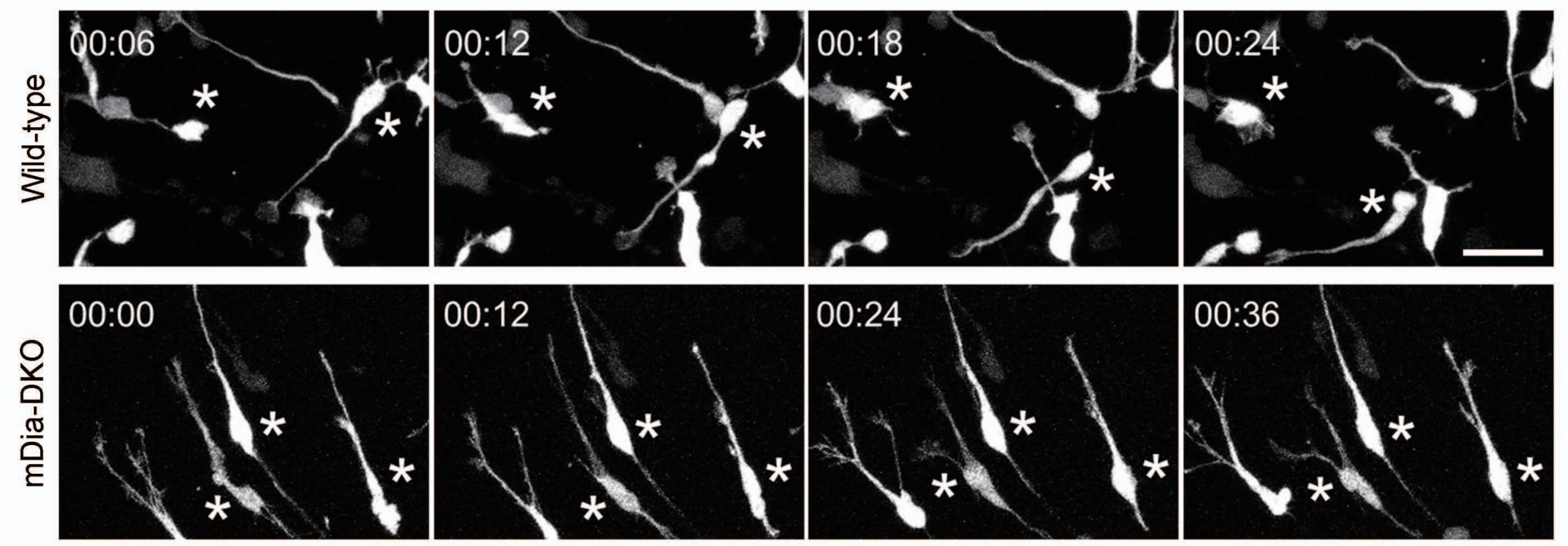

b

Wild-type

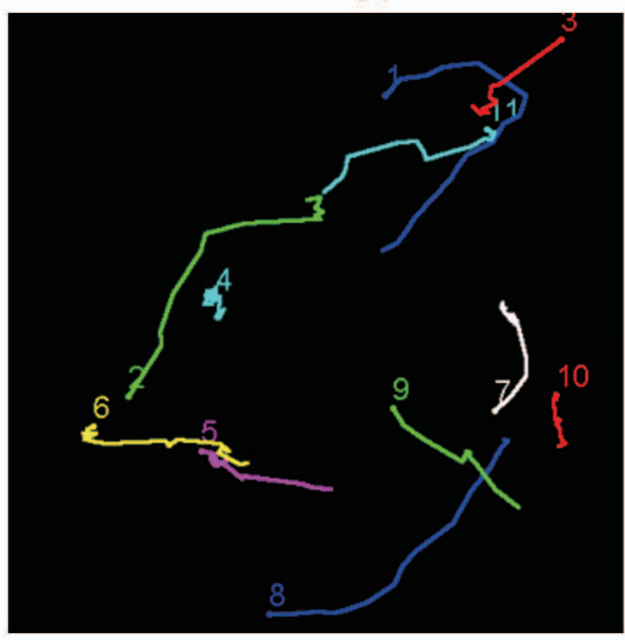

mDia-DKO

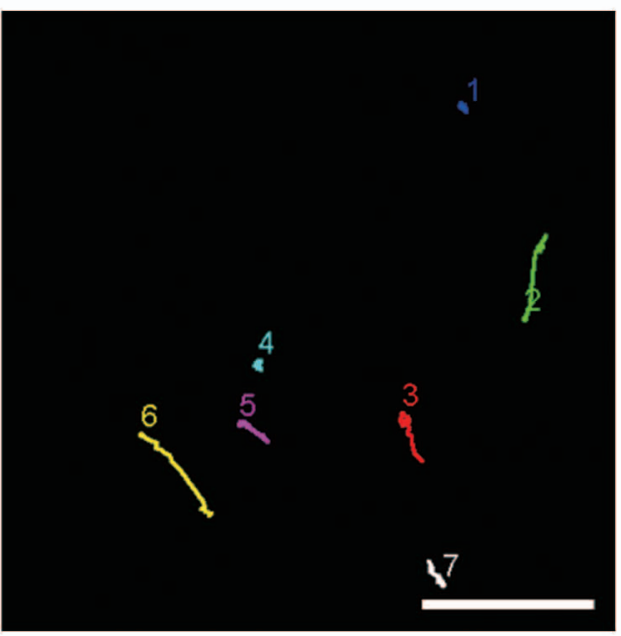

C

d
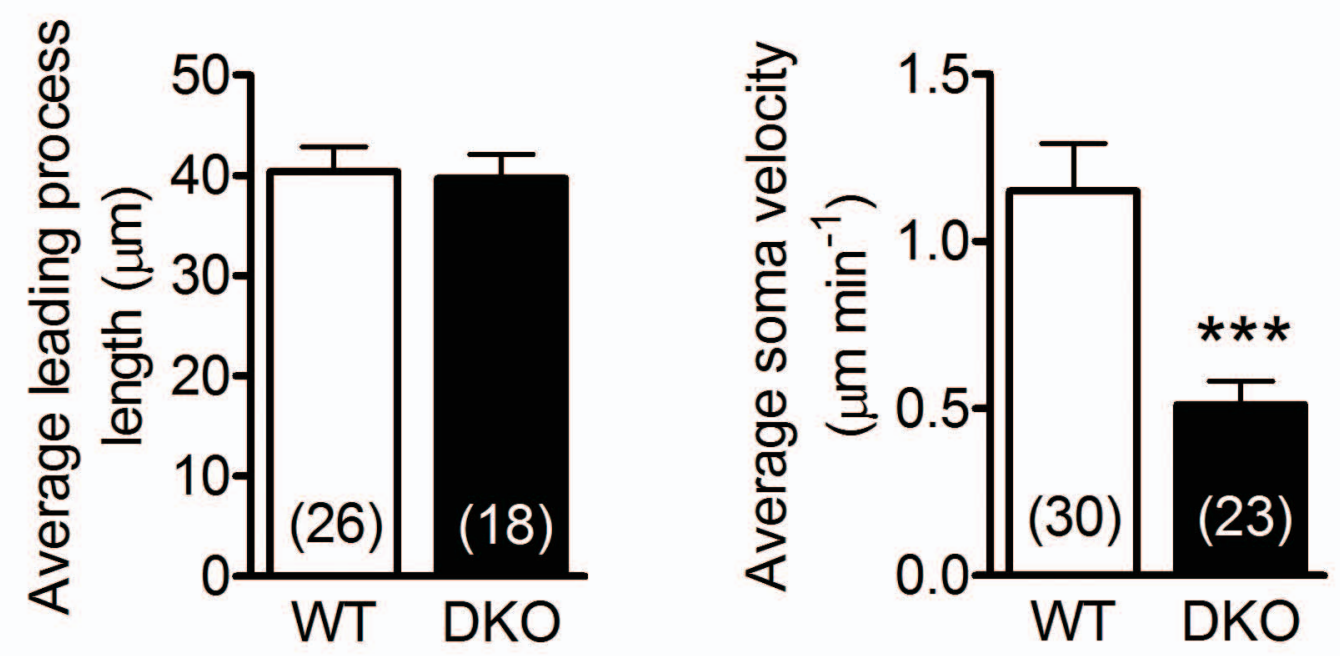

Figure 5 
a

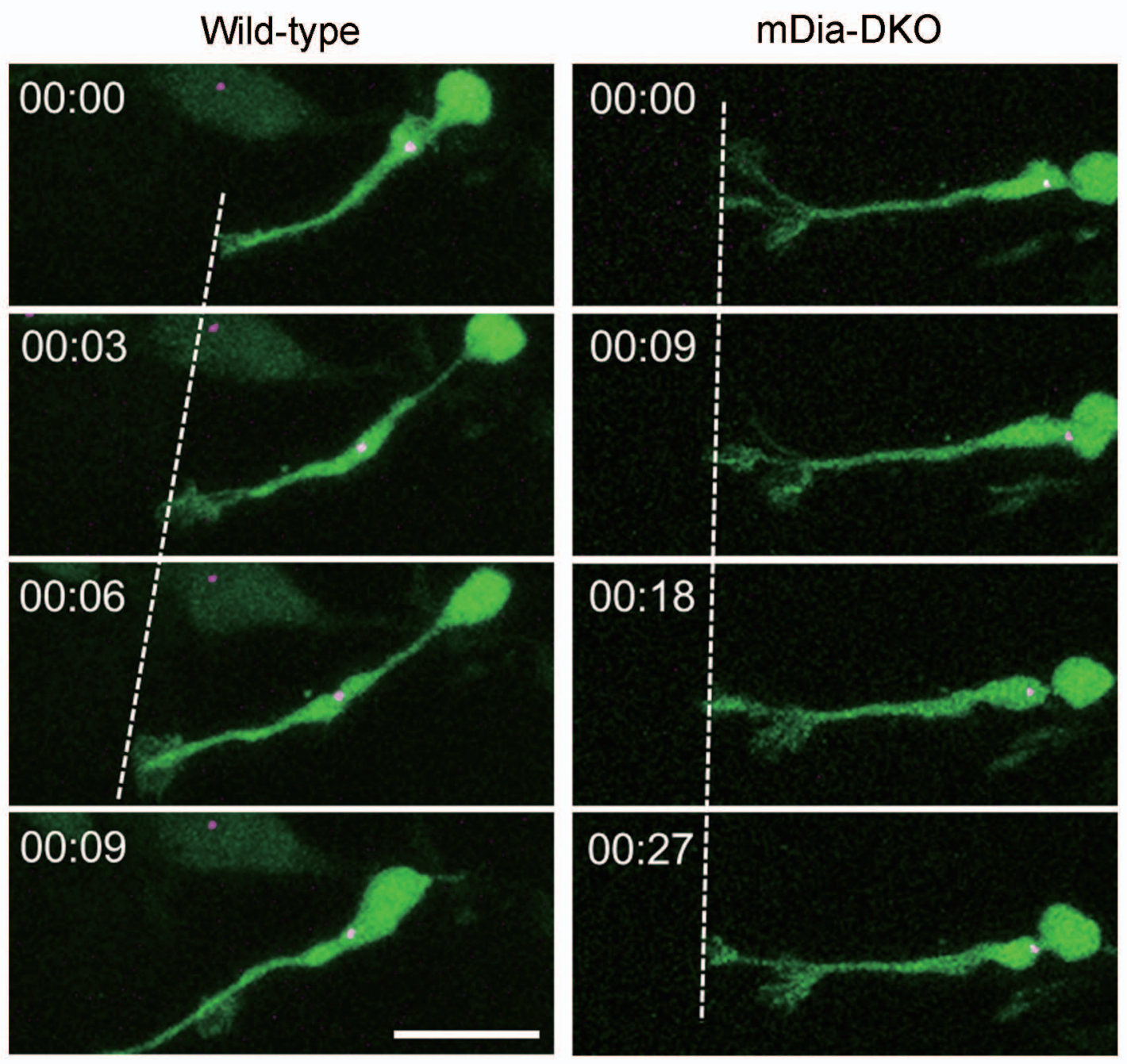

b

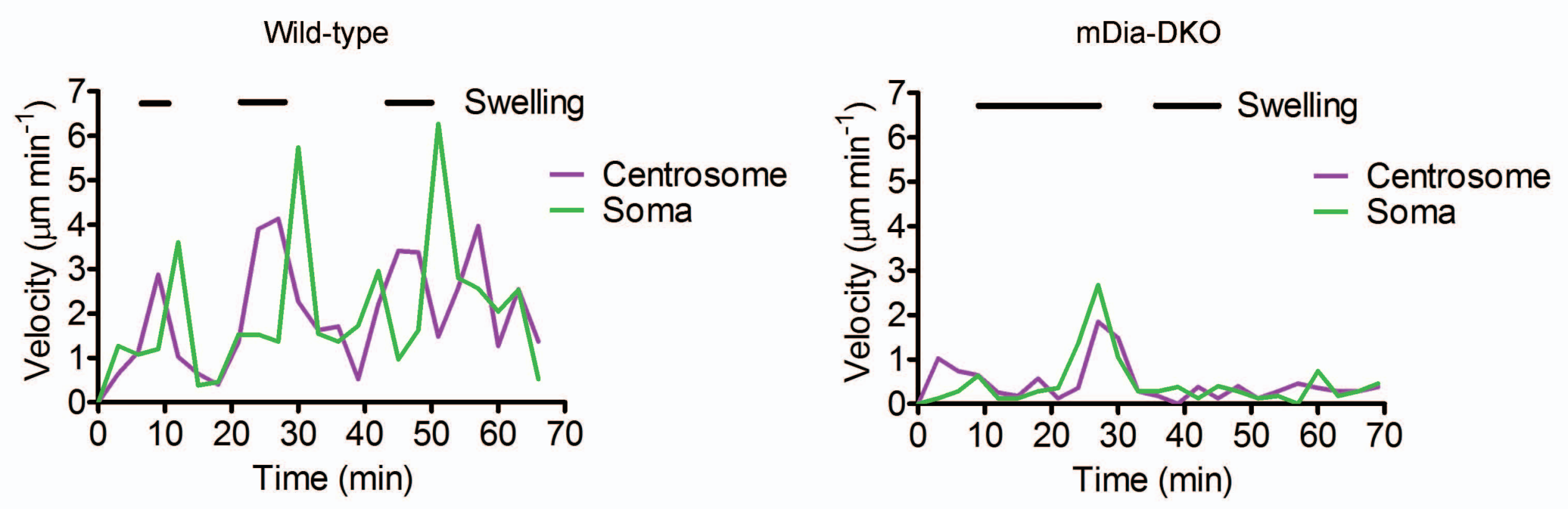

Figure 6 

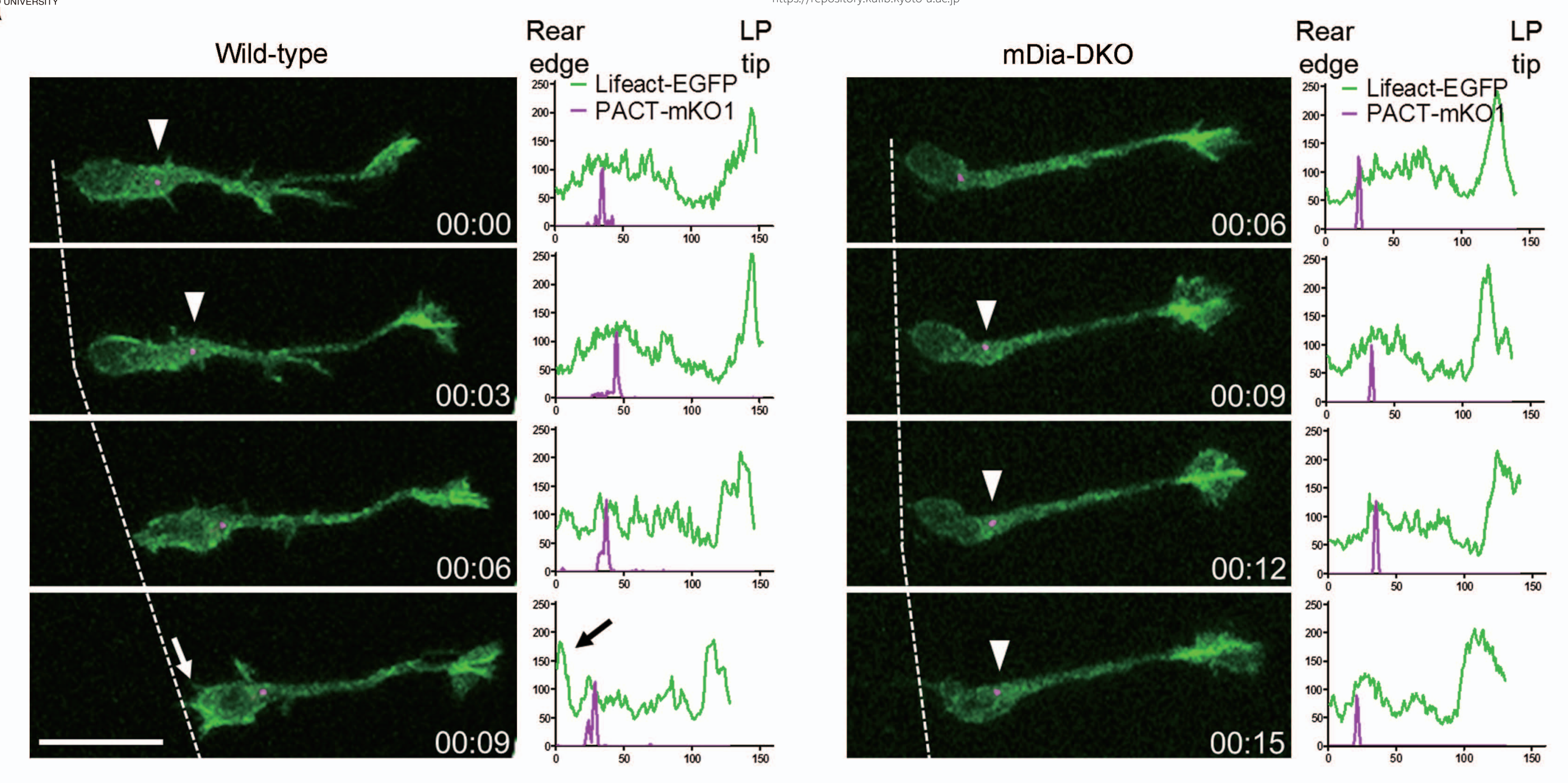

b

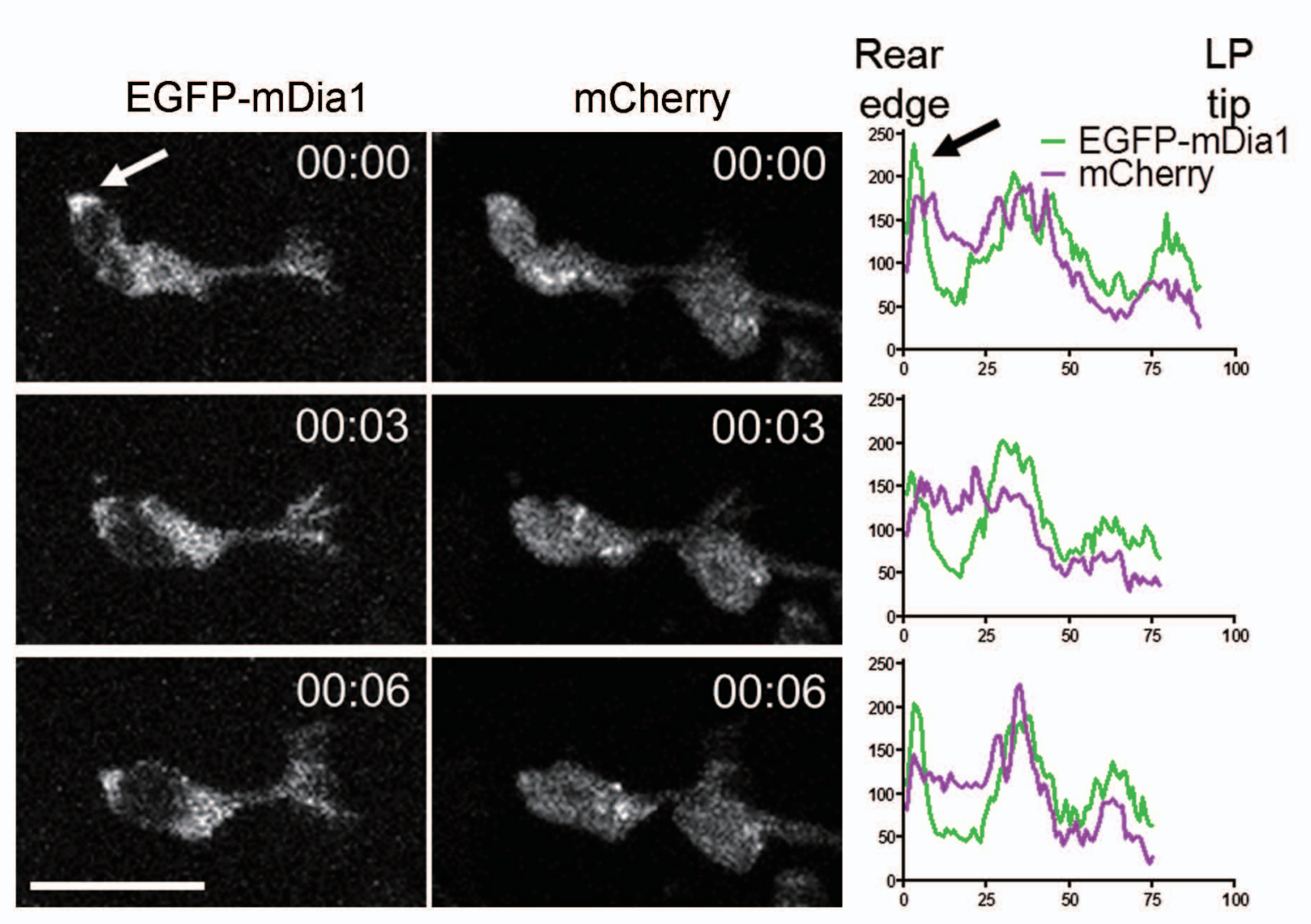

C

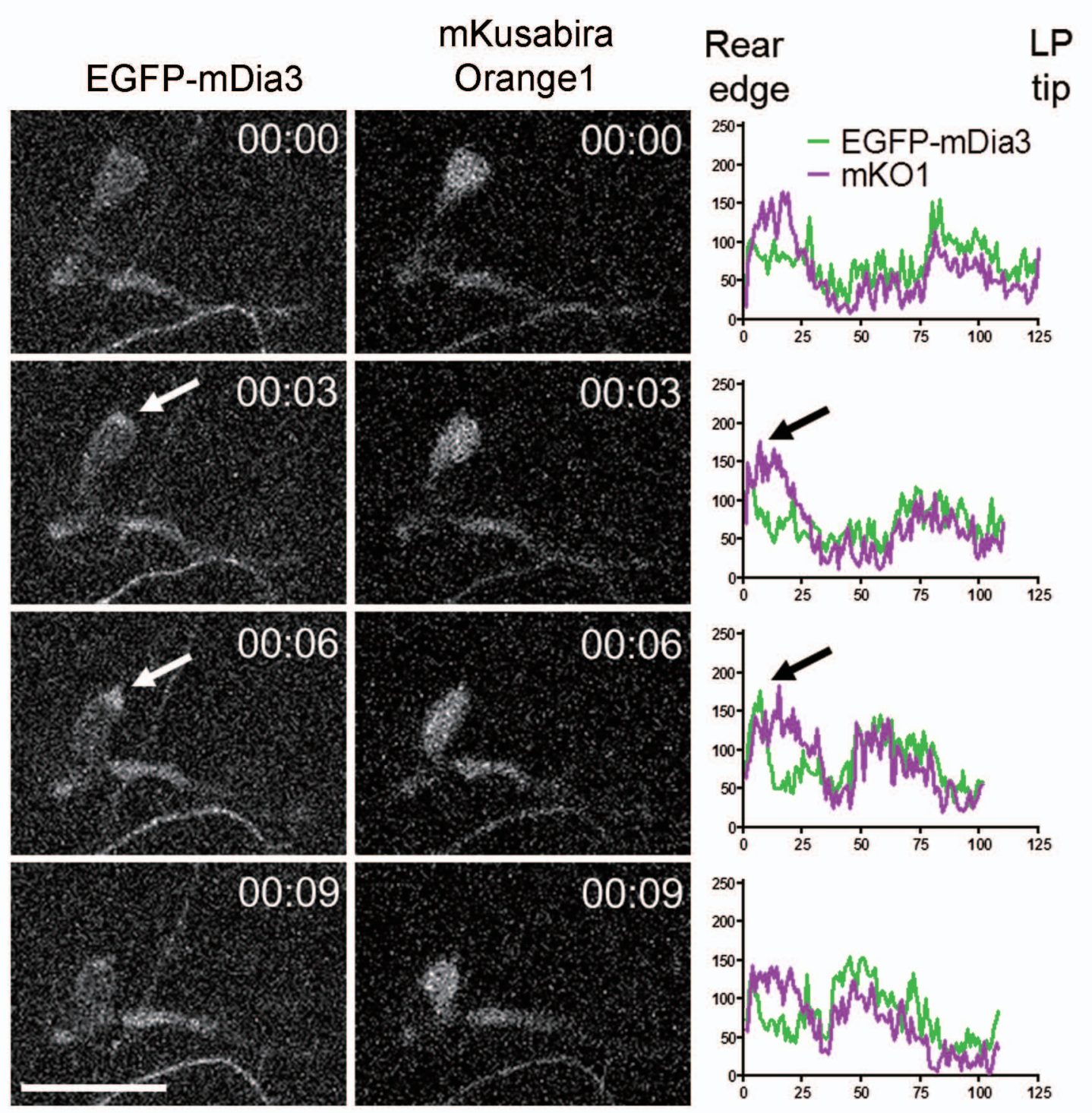

Figure 7 
a

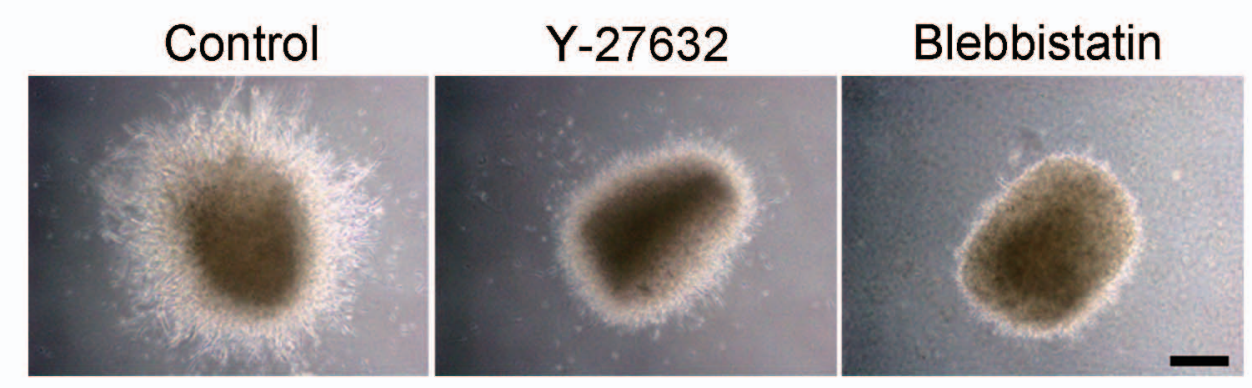

C

\section{Control}
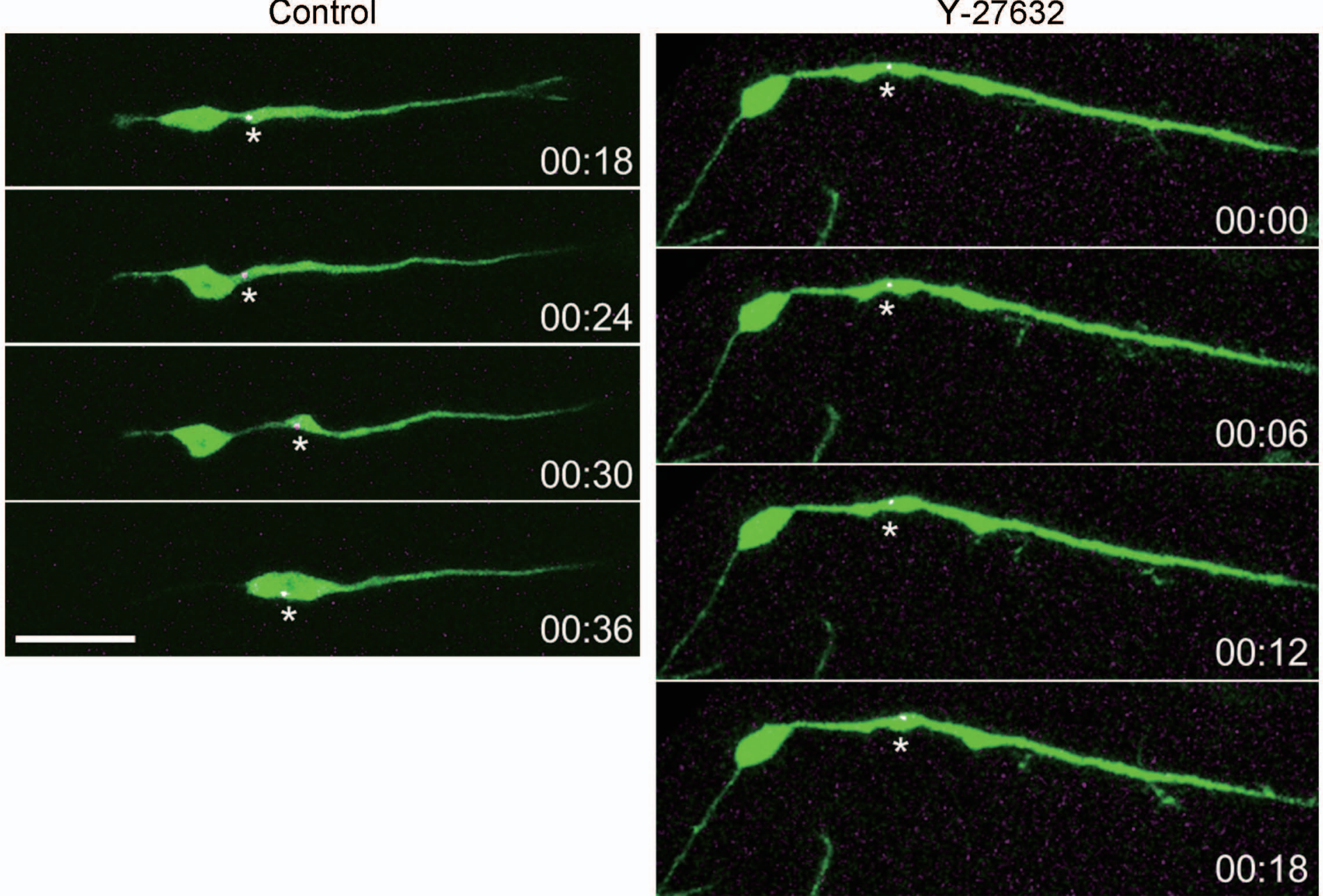

Figure 8 


\section{SUPPLEMENTARY INFORMATION}

\section{A role of $\mathbf{m D i a}$, a Rho-activated actin nucleator, in tangential migration of} interneuron precursors

Ryota Shinohara, Dean Thumkeo, Hiroshi Kamijo, Naoko Kaneko,

Kazunobu Sawamoto, Keisuke Watanabe, Hirohide Takebayashi, Hiroshi Kiyonari,

Toshimasa Ishizaki, Tomoyuki Furuyashiki, and Shuh Narumiya 

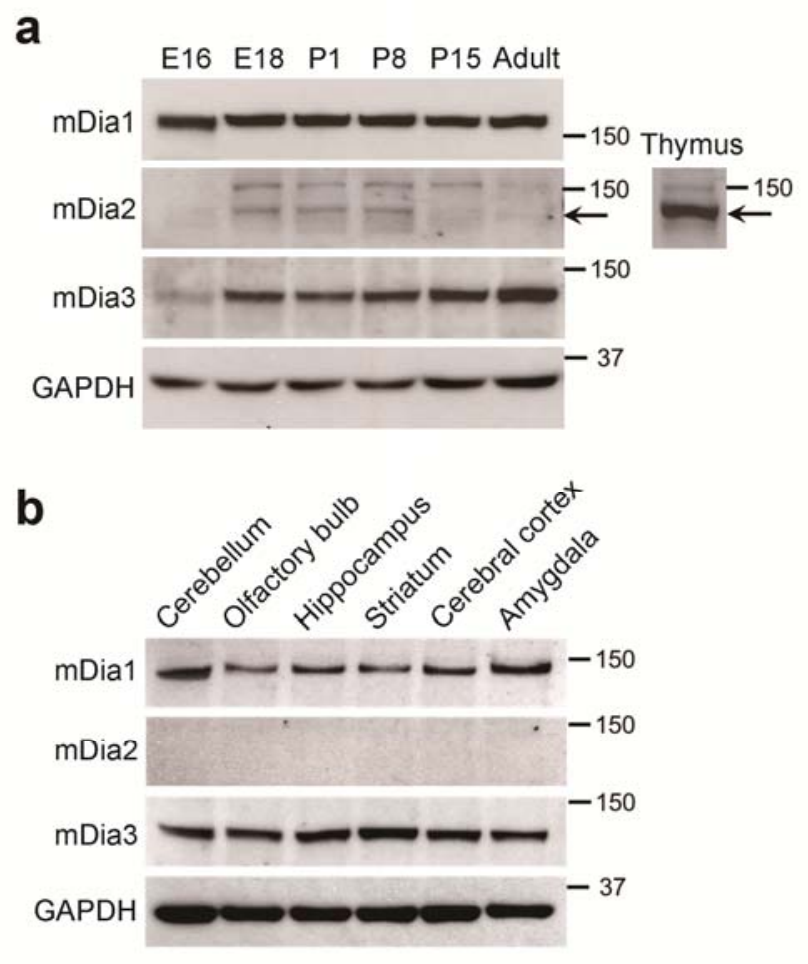

Supplementary Figure 1 Western blot analyses of mDia1 and mDia3 expression in the mouse brain.

(a) Protein expression of mDia isoforms in mouse brains at embryonic days 16 (E16), E18, postnatal days 1 (P1), P8 and P15, and in the adulthood (8-week old). Protein expression of mDia2 in the thymus is also shown as a positive control. Arrows indicate the level of mDia2 signals that are reduced by RNAi-mediated knockdown in cell lines ${ }^{1}$. GAPDH was used as an internal control. Size markers (in $\mathrm{kDa}$ ) are shown on the right. The specificity of mDia1 ${ }^{2}$ and mDia3 (Supplementary Fig. 3c) signals was confirmed using lysates from respective knockout mice. A corresponding region that the indicated protein had been blotted was taken from a membrane, and subjected to Western blotting. The level of mDia1 expression in the brain appears constant from E16 through the adulthood. In contrast, mDia3 expression was increased from a late embryonic stage towards the adulthood. mDia2 signals were detected in the brain at a low level during a perinatal period such as E18, P1, and P8.

(b) Protein expression of mDia isoforms in various regions of the adult mouse brain. A corresponding region that the indicated protein had been blotted was taken from a membrane, and subjected to Western blotting. Both mDia1 and mDia3 were expressed across all regions of the adult brain examined. 
a

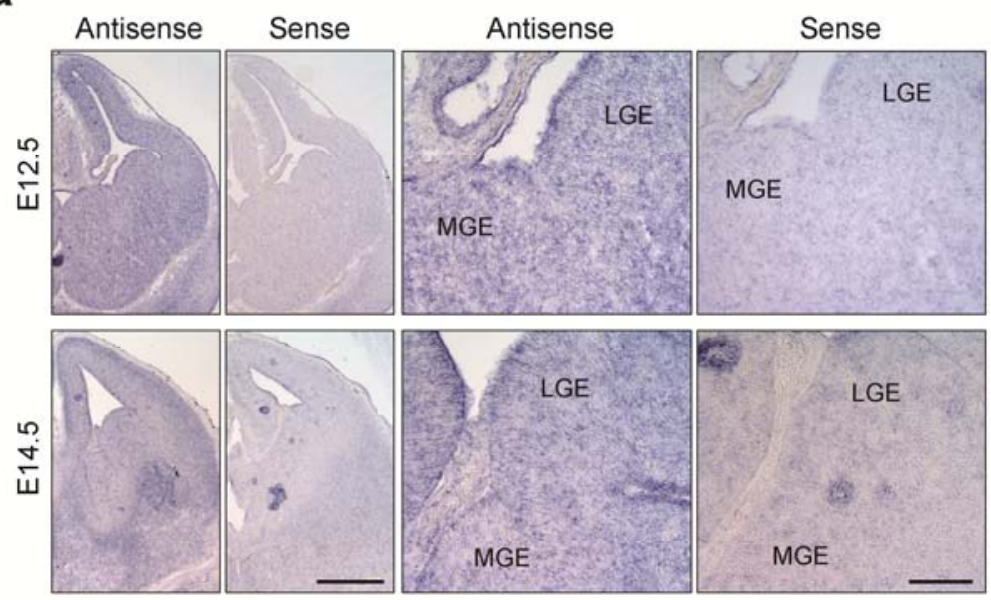

b

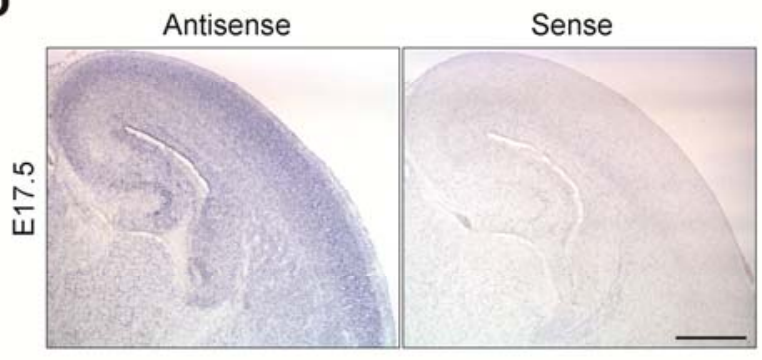

C

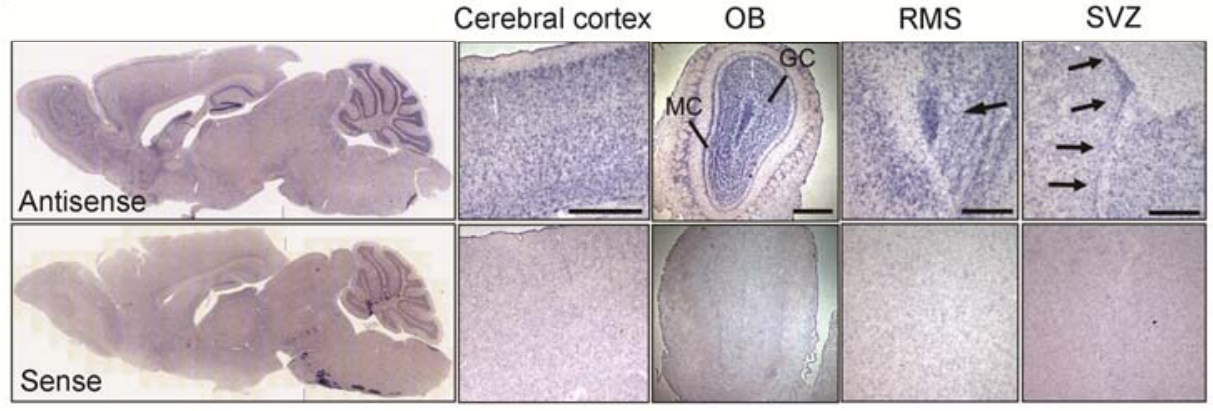

Supplementary Figure 2 Expression patterns of mDia1 in developing and adult mouse brains.

(a) In situ hybridization for mDia1 mRNA in E12.5 and E14.5 mouse brains. Antisense mDia1 riboprobes labeled with digoxigenin-UTP were used to detect mDia1 mRNA. Sense probes of the corresponding sequence were served as a negative control. Right panels are magnified images of the lateral (LGE) and medial (MGE) ganglionic eminences. mDia1 mRNA was detected across brain regions such as the developing cerebral cortex, LGE and MGE. mDia1 mRNA in the GEs was detected across the whole GEs. Scale bars, $500 \mu \mathrm{m}$ at lower magnification and $100 \mu \mathrm{m}$ at higher magnification.

(b) In situ hybridization for mDia1 mRNA in E17.5 mouse brains. Sense probes were served as a negative control. mDia1 mRNA was mainly detected in the cortical plate and the developing hippocampus. Scale bar, $500 \mu \mathrm{m}$.

(c) In situ hybridization for mDia1 mRNA in the adult mouse brain. Sense probes of the corresponding region were served as a negative control. mDia1 mRNA was detected in cells of neuron-like morphology and distribution, including neurons in the cerebral cortex and the hippocampus, and mitral and granule cells in the olfactory bulb. Positive signals were also prominent in the RMS and SVZ (arrows). OB, olfactory bulb; MC, mitral cell; GC, granule cell. Scale bars, $500 \mu \mathrm{m}$. 
a
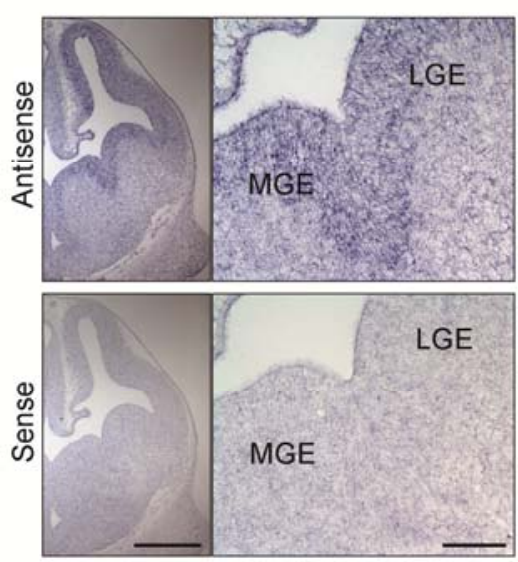

b

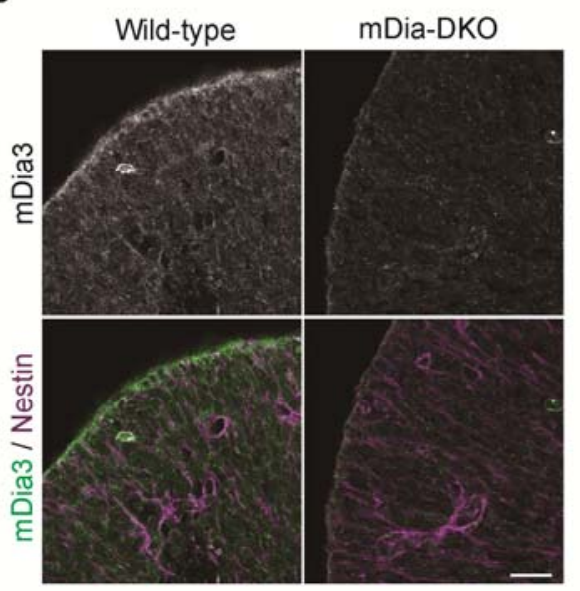

C

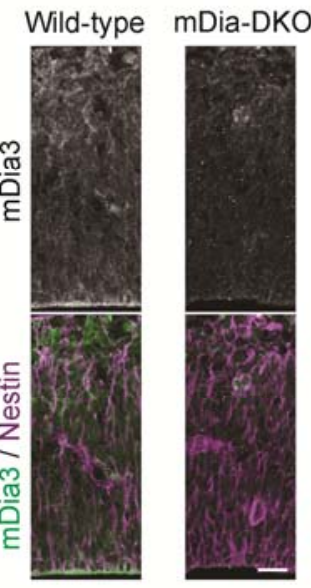

Supplementary Figure 3 Expression patterns of mDia3 in developing mouse brains.

(a) In situ hybridization for mDia3 mRNA in E12.5 mouse brains. Antisense mDia3 riboprobes labeled with digoxigenin-UTP were used to detect mDia3 mRNA. Sense probes of the corresponding sequence were served as a negative control. Right panels are magnified images of the LGE and MGE. mDia3 mRNA was widely detected in the whole brain of E12.5, though it appears to be enriched in the ventricular zone of the GEs and the developing cortex. Scale bars, $500 \mu \mathrm{m}$ at lower magnification and $100 \mu \mathrm{m}$ at higher magnification.

(b) Double immunofluorescence for mDia3 (white and green) and Nestin (purple) in the MGE at E13.5. The specificity of mDia3 signals was confirmed using brain sections from mice lacking mDia1 and mDia3 (mDia-DKO). Scale bar, $25 \mu \mathrm{m}$.

(c) Double immunofluorescence for mDia3 (white and green) and Nestin (purple) in the cerebral cortex at E13.5. The specificity of mDia3 signals was confirmed using brain sections from mDia-DKO mice. The ventricular surface is at the bottom of each image. Scale bar, $25 \mu \mathrm{m}$. 
a

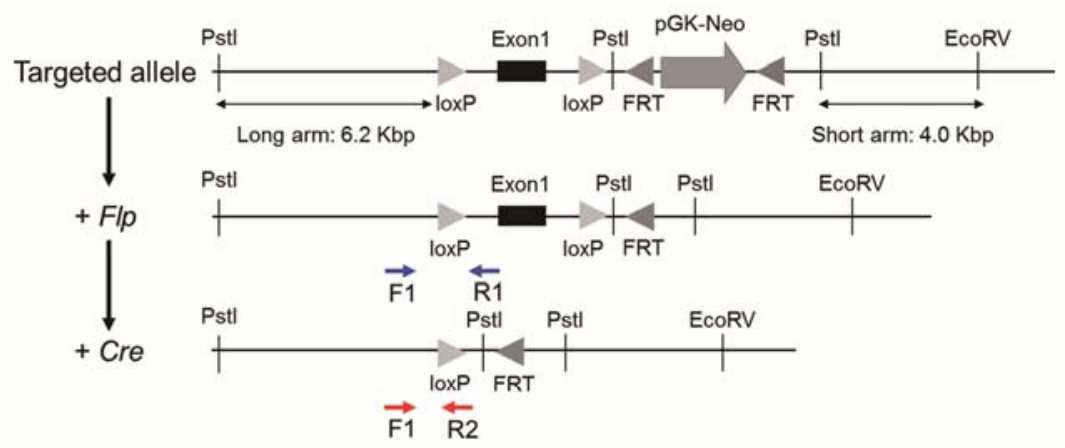

b

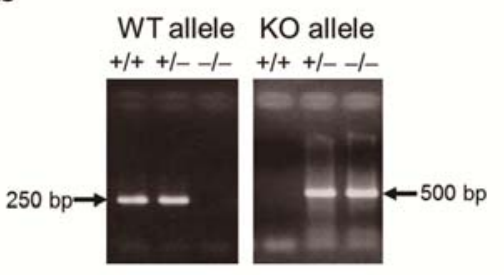

C

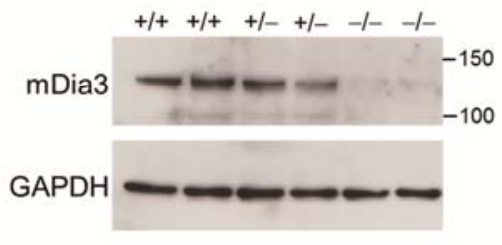

Supplementary Figure 4 Generation of mDia3-KO mice.

(a) Generation of mDia3-KO mice. Diagrams of the targeted allele, and alleles after flippase (Flp) treatment followed by Cre recombinase treatment are shown. LoxP sites flanking the first exon of mDia3 were inserted together with neomycin-resistant gene (Neo). The Neo sequence was removed by Flp-based recombination. Then, the first exon of mDia3 was deleted by ubiquitous expression of Cre recombinase to generate mDia3-KO mice. Blue and red arrows indicate the positions of primers for genomic PCR to detect the wild-type and deleted alleles, respectively (see Methods for details). Note that the diagrams are not in scale.

(b) Detection of the wild-type (WT) and deleted (KO) alleles of mDia3 by genomic PCR with primers indicated in a.

(c) mDia3 expression in the spleen lysates from wild-type $(+/+)$, heterozygous $(+/-)$, and homozygous $(-/-)$ mice of mDia3 genotype. Size markers (in $\mathrm{kDa}$ ) are shown on the right. GAPDH was used as an internal control. A corresponding region that the indicated protein had been blotted was taken from a membrane, and subjected to Western blotting. 
a

\begin{tabular}{|c|c|c|c|c|c|c|}
\hline & \multicolumn{3}{|c|}{ E15-18 } & \multicolumn{3}{c|}{3 weeks } \\
\hline mDia1 & $+/+$ & $+/-$ & $-/-$ & $+/+$ & $+/-$ & $-/-$ \\
\hline mDia3 & null & null & null & null & null & null \\
\hline Offspring & 50 & 124 & 49 & 165 & 258 & 27 \\
\hline Observation (\%) & 22.4 & 55.6 & 22.0 & 36.7 & 57.3 & 6.0 \\
\hline Expectation (\%) & 25 & 50 & 25 & 25 & 50 & 25 \\
\hline
\end{tabular}

b

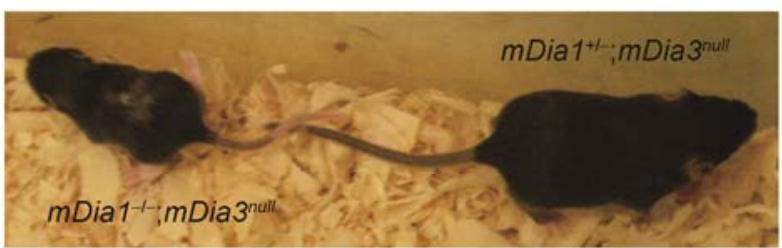

C

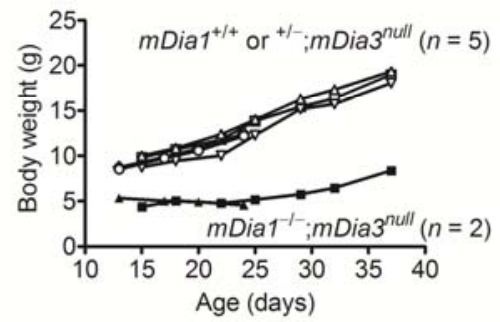

d

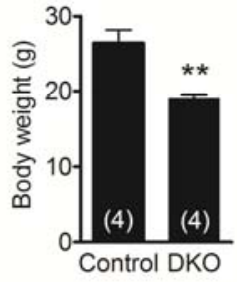

Supplementary Figure 5 mDia-DKO mice show decreased body size, growth retardation and a high mortality rate after birth.

(a) The proportions of mDia1 wild-type $(+/+)$, heterozygous $(+/-)$ and homozygous (/-) $\mathrm{mDia}^{\text {null }}$ offsprings by crossing $\mathrm{mDia1}^{+/-} ; \mathrm{mDia}^{-/ \mathrm{y}}$ male and $\mathrm{mDia1}^{+/-} ; \mathrm{mDia}^{-/-}$ female at E15-18 and 3 weeks after birth. Expected proportions of respective genotypes based on Mendelian frequency are also shown at the bottom. Note that mDia-DKO mice were observed close to the Mendelian frequency at E15-18, three-fourths of them died during the first three weeks after birth.

(b) General appearance of female littermates at P15 of mDia-DKO ( $\mathrm{mDia}^{-/-} ; \mathrm{mDia}^{\text {null }}$, shown in left) and control $\left(m D i a 1^{+/-} ; m D i a 3^{\text {null }}\right.$, shown in right) mice. mDia-DKO mice were considerably smaller than control mice. Also note that the coat color was partially lost at the back of this mDia-DKO mouse.

(c) Body weight gain after birth in representative $m D i a 3^{\text {null }}$ littermates of various $m D i a 1$ genotypes. Note that two individuals of mDia-DKO mice $\left(\mathrm{mDia1}^{-1-} ; \mathrm{mDia}^{\text {null }}\right)$ showed reduced body weight gain after birth, compared with the other genotypes (mDia1 ${ }^{+/+}$or ${ }^{+-} ;$mDia3 ${ }^{\text {null }}$ ).

(d) Body weights of 12-13 week-old mDia3-KO and mDia-DKO female littermates. The numbers of mice are shown in each column. ${ }^{* *} P<0.01, t$-test. Error bars represent s.e.m. 
a

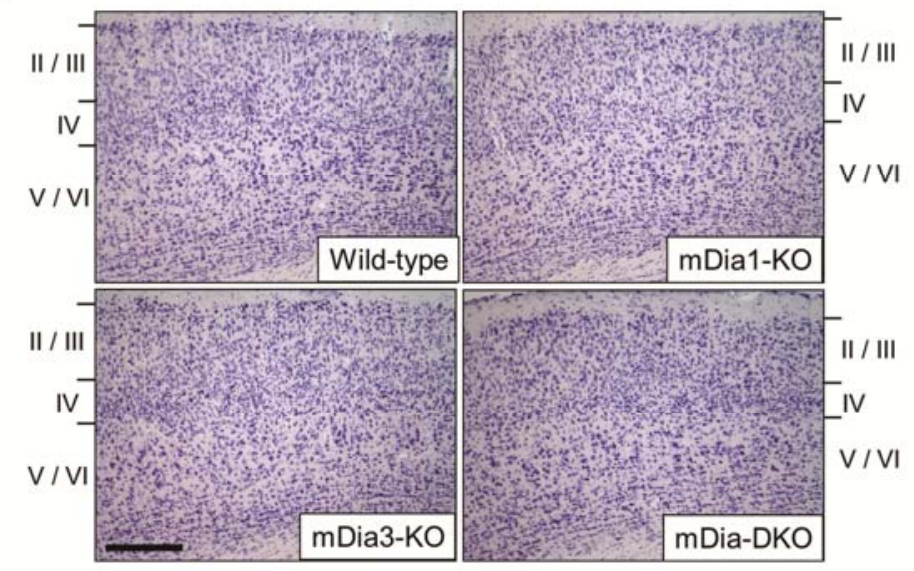

b
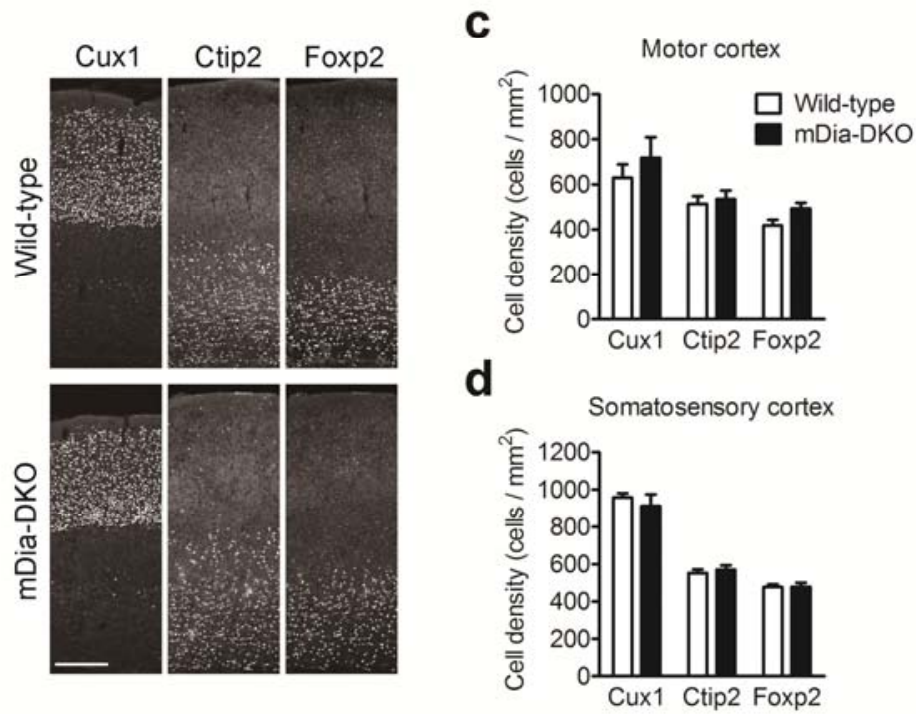

Supplementary Figure 6 The layer formation of cortical excitatory neurons is not impaired in mDia-DKO mice.

(a) Nissl staining of coronal sections of the somatosensory cortex from adult wild-type, mDia1-KO, mDia3-KO, and mDia-DKO mice. Layer II/III, layer IV, and layer V/VI are clearly observed in all of these genotypes. Scale bar, $250 \mu \mathrm{m}$.

(b) Immunofluorescence for cortical layer-specific markers (Cux1, Ctip2, and Foxp2) in coronal sections of the somatosensory cortex from adult wild-type and mDia-DKO mice. mDia-DKO mice display a similar distribution of excitatory neurons labeled with these markers compared to wild-type mice. Scale bar, $250 \mu \mathrm{m}$.

(c) Quantification of the densities of Cux1, Ctip2, or Foxp2-positive cells in the motor and somatosensory cortex of adult wild-type and mDia-DKO mice $(n=4$ mice for each genotype). Error bars represent s.e.m. 
a

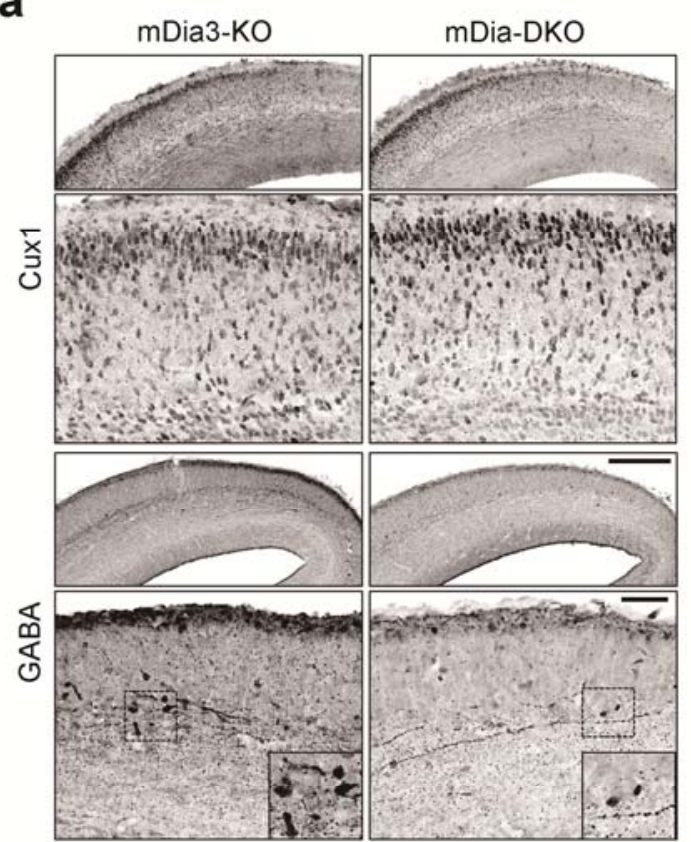

b

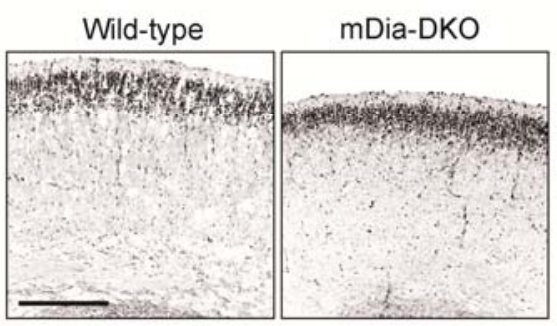

Supplementary Figure 7 Immunofluorescence for Cux1 and GABA in the developing cerebral cortex of mDia-DKO mice.

(a) Immunofluorescence for Cux1 and GABA in coronal sections of the cerebral cortex from mDia3-KO and mDia-DKO littermates at E16.5. Insets in the bottom panels are magnified views of GABA-containing cells of each genotype. Signals are shown in gray scale to improve the visibility. Scale bars, $250 \mu \mathrm{m}$ at lower magnification and $50 \mu \mathrm{m}$ at higher magnification.

(b) Immunofluorescence for Cux1 in coronal sections of the cerebral cortex from P0 wild-type and mDia-DKO mice. mDia-DKO mice display a similar distribution of Cux1-positive cells compared to wild-type mice. Signals are shown in gray scale to improve visibility. Scale bar, $250 \mu \mathrm{m}$. 


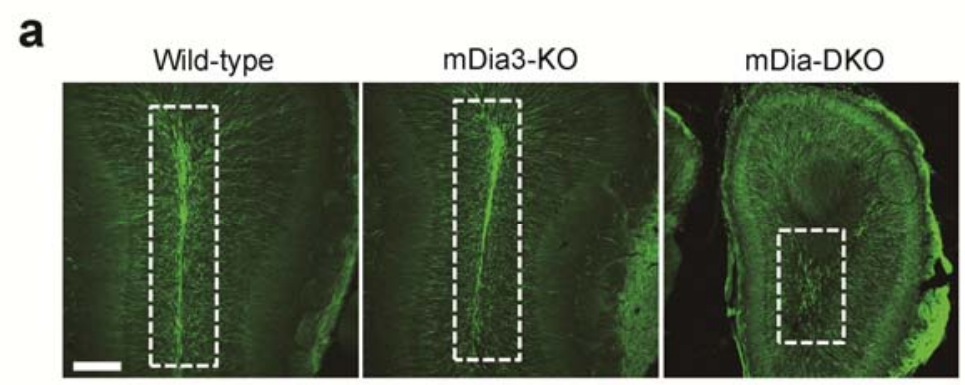

b

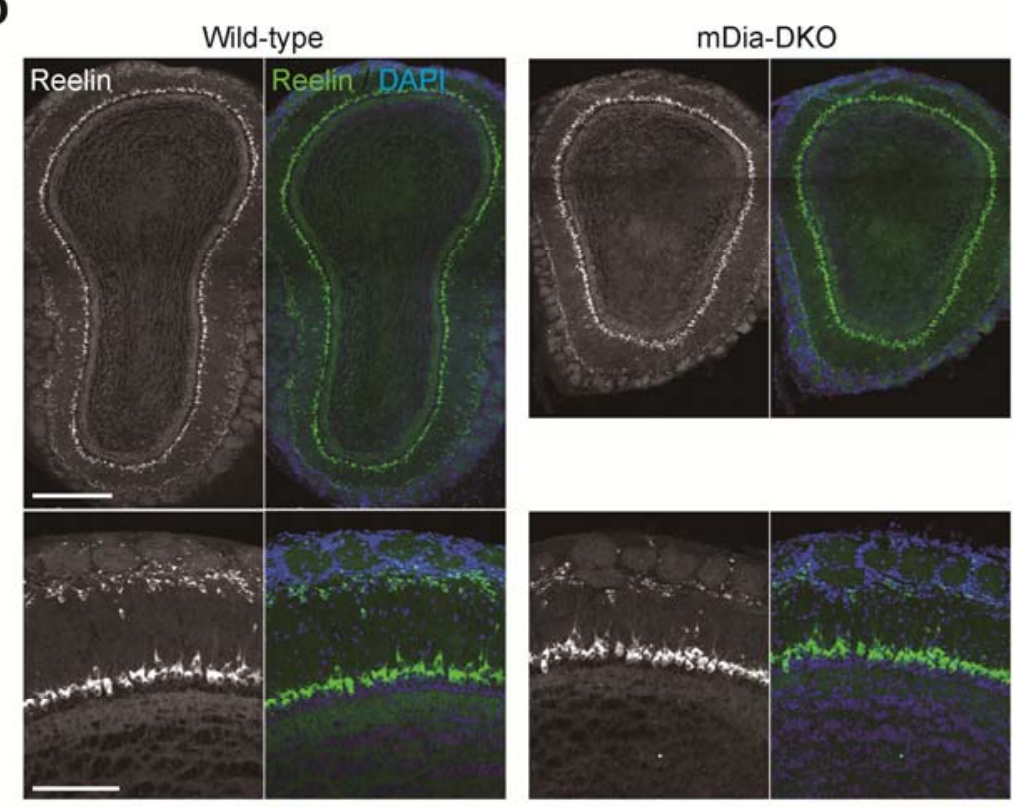

C

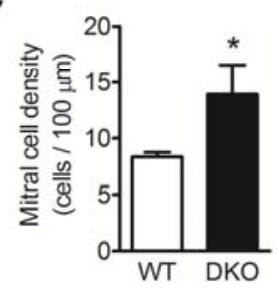

Supplementary Figure 8 Immunofluorescence for doublecortin and Reelin in the olfactory bulb of adult mDia-DKO mice.

(a) Immunofluorescence for doublecortin in coronal sections in the olfactory bulb of adult wild-type, mDia3-KO, and mDia-DKO mice. Dotted rectangles indicate the RMS. Scale bar, $250 \mu \mathrm{m}$.

(b) Immunofluorescence for Reelin (white and green) and nuclear counterstaining with DAPI (blue) in the olfactory bulb of adult wild-type and mDia-DKO mice. Cells expressing Reelin, a marker for mitral cells, are similarly found in the olfactory bulb of wild-type and mDia-DKO mice. Scale bars, $500 \mu \mathrm{m}$ at lower magnification and $150 \mu \mathrm{m}$ at higher magnification.

(c) The density of Reelin-positive mitral cells in the olfactory bulb of adult wild-type and mDia-DKO mice. Eight sections from 3 wild-type mice and 6 sections from 2 mDia-DKO mice were used, and the number of cells in randomly chosen $500 \mu \mathrm{m}$ perimeter was counted. $* P<0.05$, $t$-test. Error bars represent s.e.m. 


\section{a}

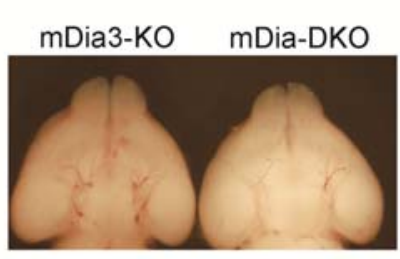

b

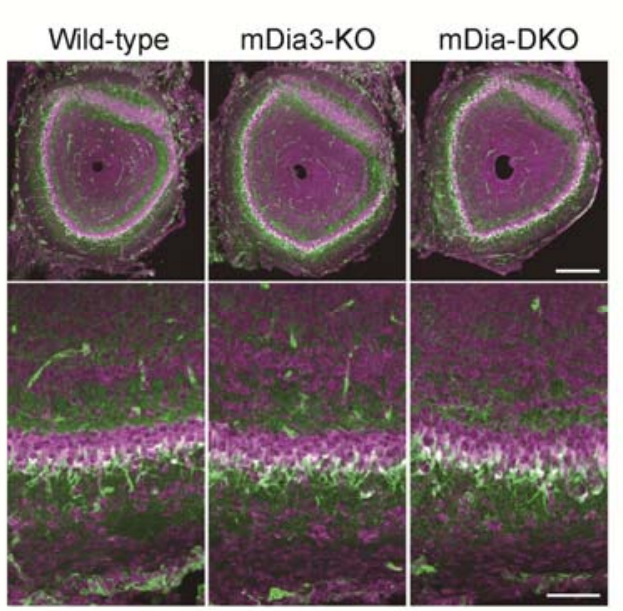

Supplementary Figure 9 Gross appearance of the brain and the layer formation of mitral cells in the olfactory bulb of E18.5 embryos of various mDia genotypes.

(a) Gross appearance of the brain of mDia3-KO and mDia-DKO mice at E18.5.

(b) Immunofluorescence for Reelin (green) and Nissl staining (purple) in the olfactory bulb of wild-type, mDia3-KO and mDia-DKO mice at E18.5. Scale bars, $200 \mu \mathrm{m}$ at lower magnification and $50 \mu \mathrm{m}$ at higher magnification. 

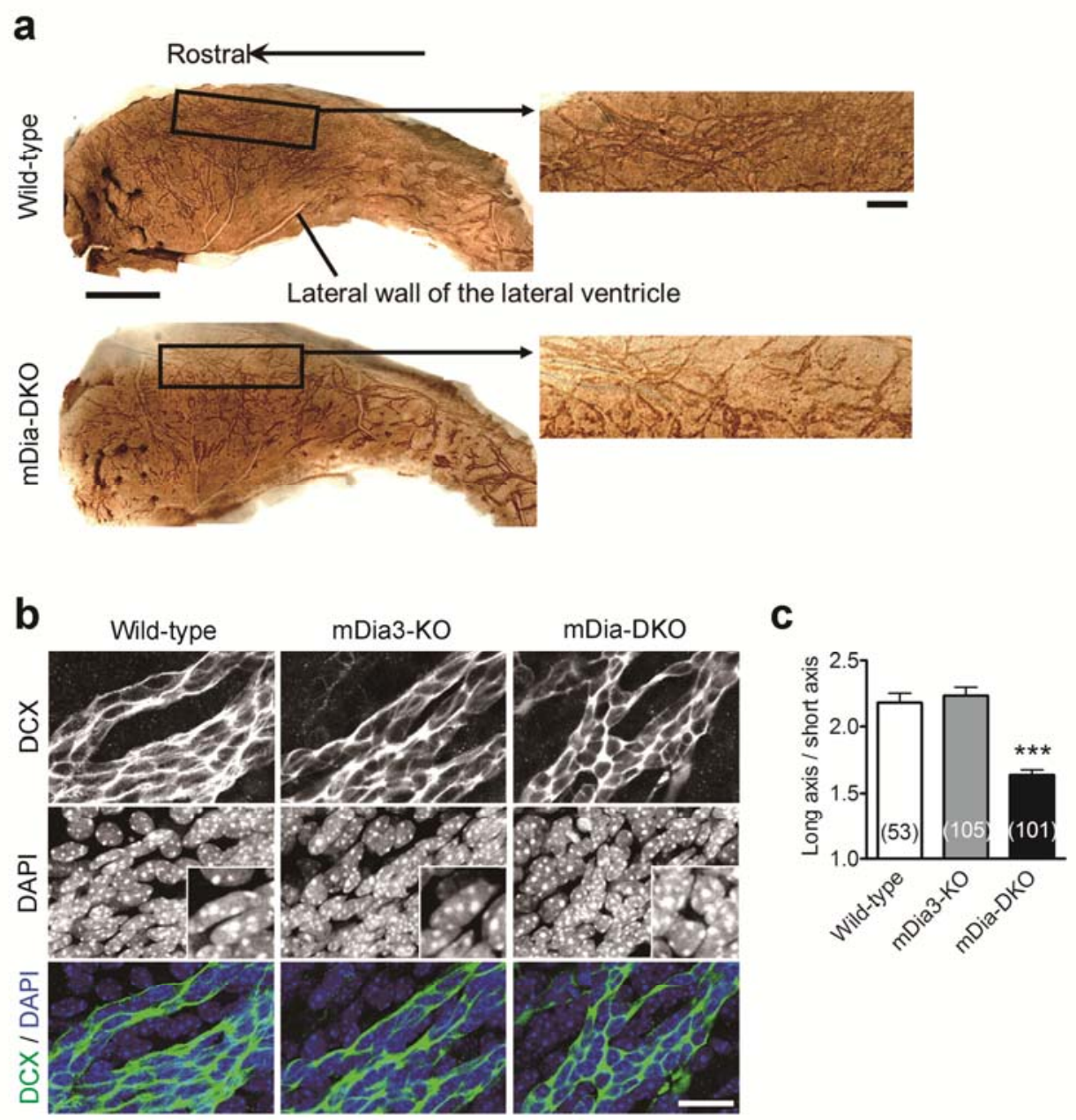

Supplementary Figure 10 Defective migration of SVZ neuroblasts on the lateral wall of the lateral ventricle of mDia-DKO mice.

(a) Whole-mount immunohistochemistry of doublecortin-positive neuroblasts on the lateral walls of the lateral ventricles from wild-type and mDia-DKO mice. Regions enclosed by rectangles are magnified and shown on the right side. Note that long neuroblast chains parallel to the dorsal ridge of the lateral ventricle observed in wild-type mice were largely absent in mDia-DKO mice. Scale bars, $500 \mu \mathrm{m}$ in the left panels and $100 \mu \mathrm{m}$ in the right panels.

(b) Whole-mount immunofluorescence of doublecortin (DCX)-positive neuroblasts on the lateral walls of lateral ventricles from wild-type, mDia3-KO, and mDia-DKO mice. Nuclei were counterstained with DAPI (blue). Insets in the middle panels are magnified views of the nuclear staining. Scale bar, $20 \mu \mathrm{m}$.

(c) Morphometric analysis of nuclei of doublecortin-positive neuroblasts in wild-type, mDia3-KO and mDia-DKO mice. The number of cells is shown in each column. Note that nuclei of mDia-DKO neuroblasts were less elongated than those of wild-type and mDia3-KO neuroblasts. ${ }^{* * *} P<0.001$, Bonferroni's post hoc test in comparison with the wild-type value following one-way $\operatorname{ANOVA}\left(F_{2,256}=34.67, P<0.0001\right)$. Error bars represent s.e.m. 


\section{a}

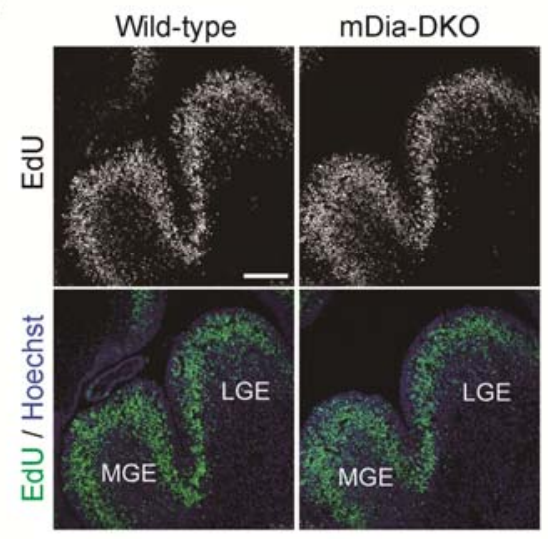

b

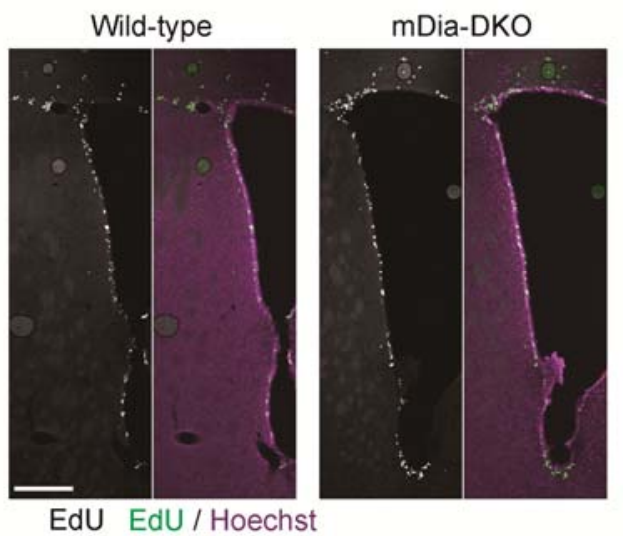

Supplementary Figure 11 Intact proliferation of neural stem and progenitor cells in mDia-DKO mice.

(a) Pulse-labeling of neural stem and progenitor cells with EdU in wild-type and mDia-DKO embryos. EdU was injected into pregnant mice carrying embryos of E13.5, and embryos were sacrificed one hour later to visualize EdU-labeled cells. EdU signals and nuclear staining with Hoechst are shown in green and blue, respectively, in merged images. LGE, lateral ganglionic eminence; MGE, medial ganglionic eminence. Scale bar, $150 \mu \mathrm{m}$.

(b) Pulse-labeling of neural stem and progenitor cells with EdU in wild-type and mDia-DKO mice. EdU was injected into adult mice, and mice were sacrificed one hour later to visualize EdU-labeled cells. EdU signals and nuclear staining with Hoechst are shown in green and purple, respectively. Scale bar, $250 \mu \mathrm{m}$. 
a

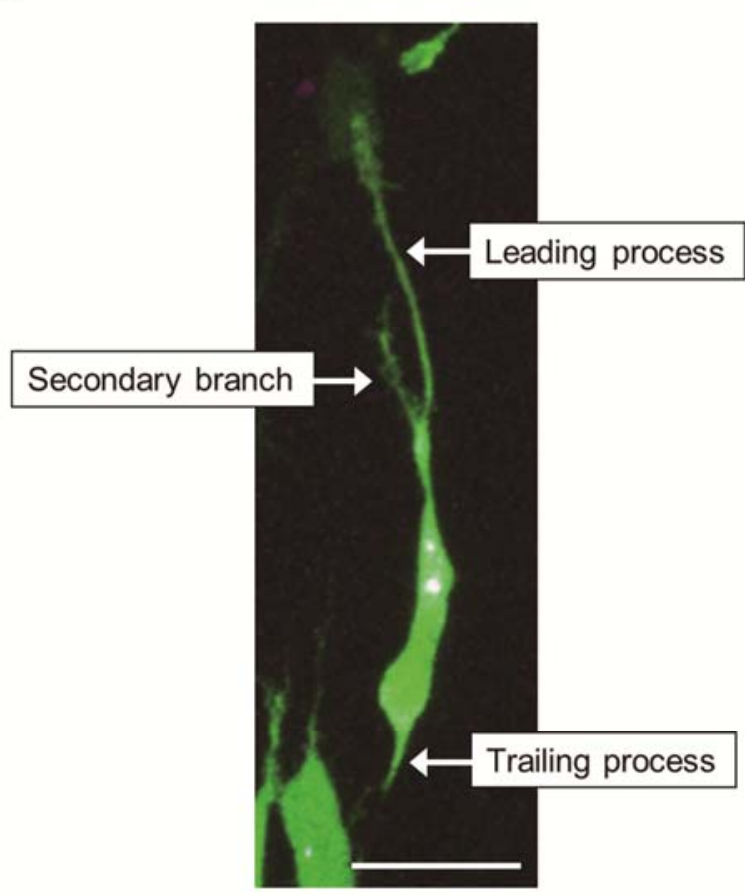

b

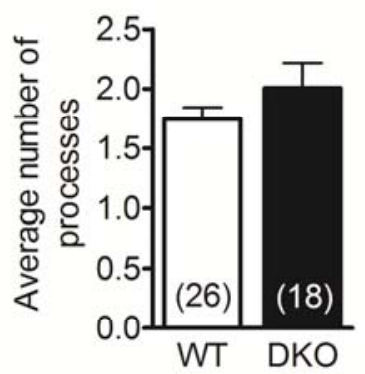

C

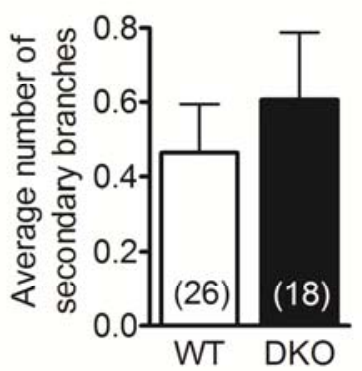

d

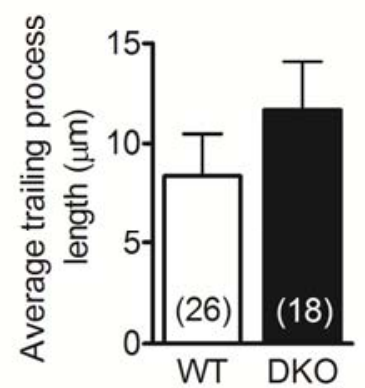

Supplementary Figure 12 Morphology of wild-type and mDia-DKO SVZ neuroblasts. (a) An example of an EGFP and PACT-mKO1-labeled, wild-type SVZ neuroblast with a secondary branch and a trailing process. Signals of EGFP and PACT-mKO1, a marker for the centrosome, are shown in green and purple, respectively. Scale bar, $20 \mu \mathrm{m}$.

(b) The average number of processes of wild-type (WT) and mDia-DKO (DKO) neuroblasts. The total number of processes including a leading process, secondary branches and a trailing process were analyzed and averaged across 10 successive images taken at 3 min intervals (see Methods).

(c) The average number of secondary branches of wild-type (WT) and mDia-DKO (DKO) neuroblasts. The number of secondary branches was averaged across 10 successive images taken at 3 min intervals.

(d) The length of a trailing process of wild-type (WT) and mDia-DKO (DKO) neuroblasts. The trailing process length was averaged from 10 successive images taken at 3 min intervals.

The number of cells is shown in each column. Error bars represent s.e.m. 
a
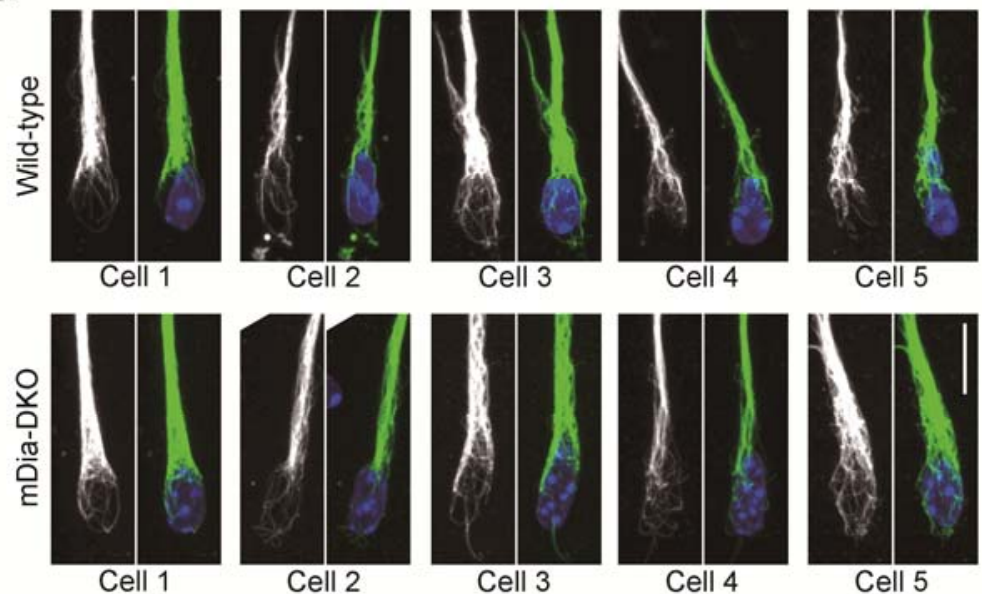

b
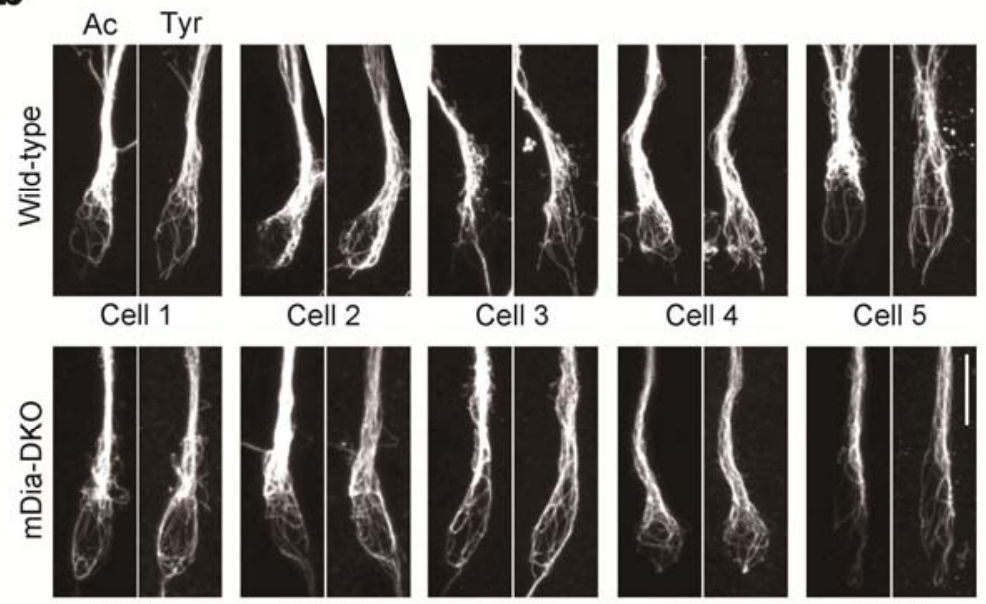

Cell 3

Cell 4

Cell 5

Cell 1
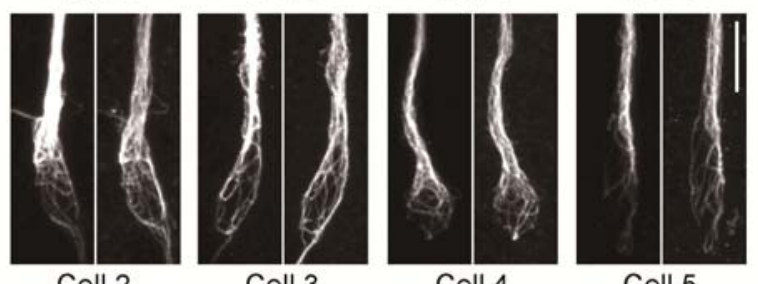

Cell 5

Supplementary Figure 13 Perinuclear microtubule structure and dynamics are not apparently altered in mDia-DKO neuroblasts.

(a) Immunofluorescence for $\alpha$-tubulin (white and green) in wild-type and mDia-DKO neuroblasts. Since antibodies used for tubulin immunostaining caused high background in three-dimensional culture in Matrigel, we applied two-dimensional culture of SVZ neuroblasts for this purpose. Nuclei were counterstained with DAPI (blue). Five representative neuroblasts out of 17 neuroblasts analyzed for each genotype are shown. Scale bar, $10 \mu \mathrm{m}$.

(b) Immunofluorescence for Acetylated tubulin (Ac) and Tyrosinated tubulin (Tyr) in the perinuclear microtubule cage in wild-type and mDia-DKO neuroblasts cultured on two-dimensional Matrigel layer. Five representative neuroblasts out of 15 neuroblasts analyzed for each genotype are shown. Scale bar, $10 \mu \mathrm{m}$. 


\section{a}

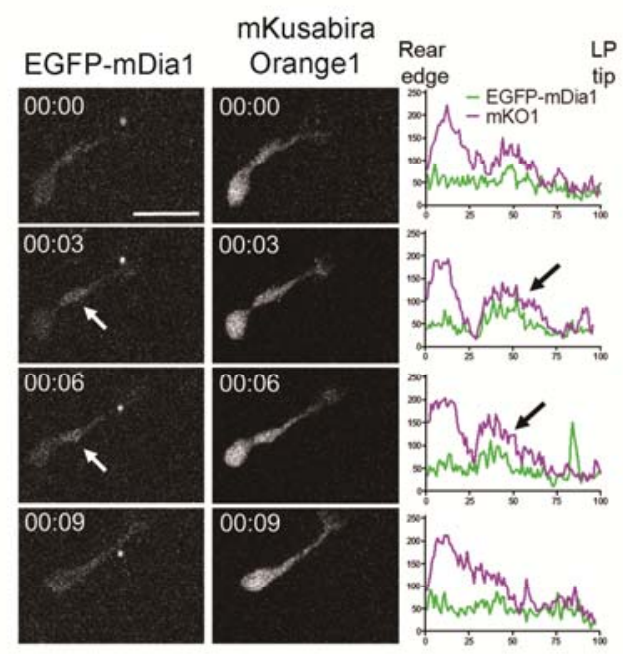

b

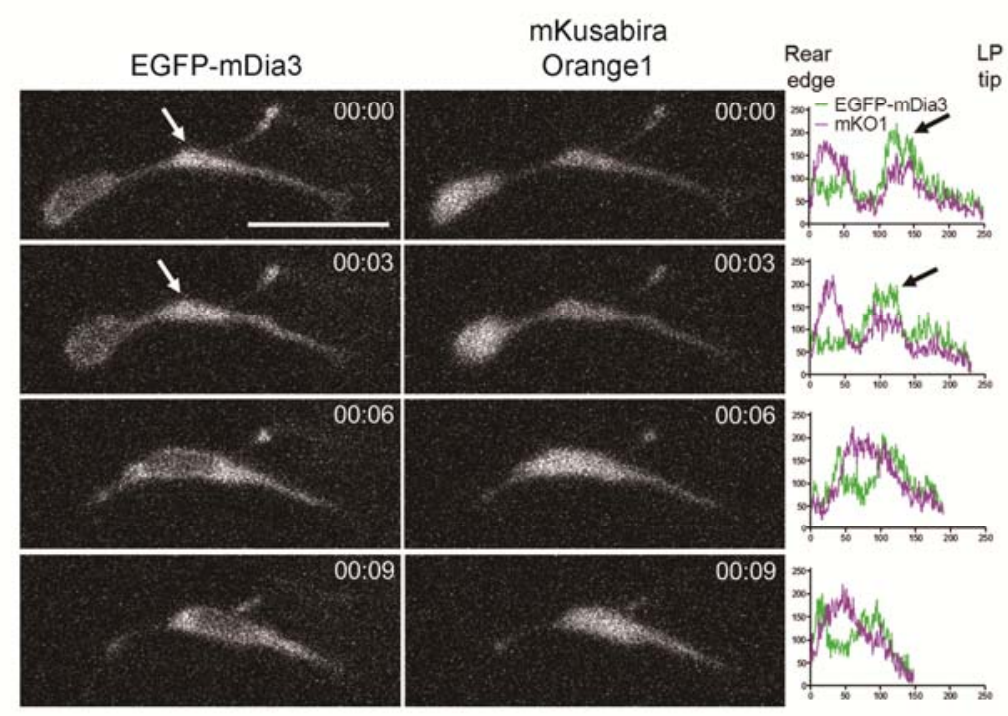

Supplementary Figure 14 Analysis of localization of mDia1 and mDia3 in migrating SVZ neuroblasts.

(a,b) Accumulation of EGFP-mDia1 (a) and EGFP-mDia3 (b) in the swelling of wild-type neuroblasts. mKusabira Orange1 (mKO1) was simultaneously visualized to monitor the distribution of the cytoplasmic bulk. Fluorescent images were acquired at 3 min intervals indicated by the number in each frame. Linescan profiles show the signal intensities of EGFP-fused mDia1 or mDia3 (green lines) and mKO1 (purple lines) from the rear edge to the tip of the leading process (LP) in the corresponding images. Arrows indicate the accumulation of EGFP-mDia in the swelling. Scale bar, $20 \mu \mathrm{m}$. 


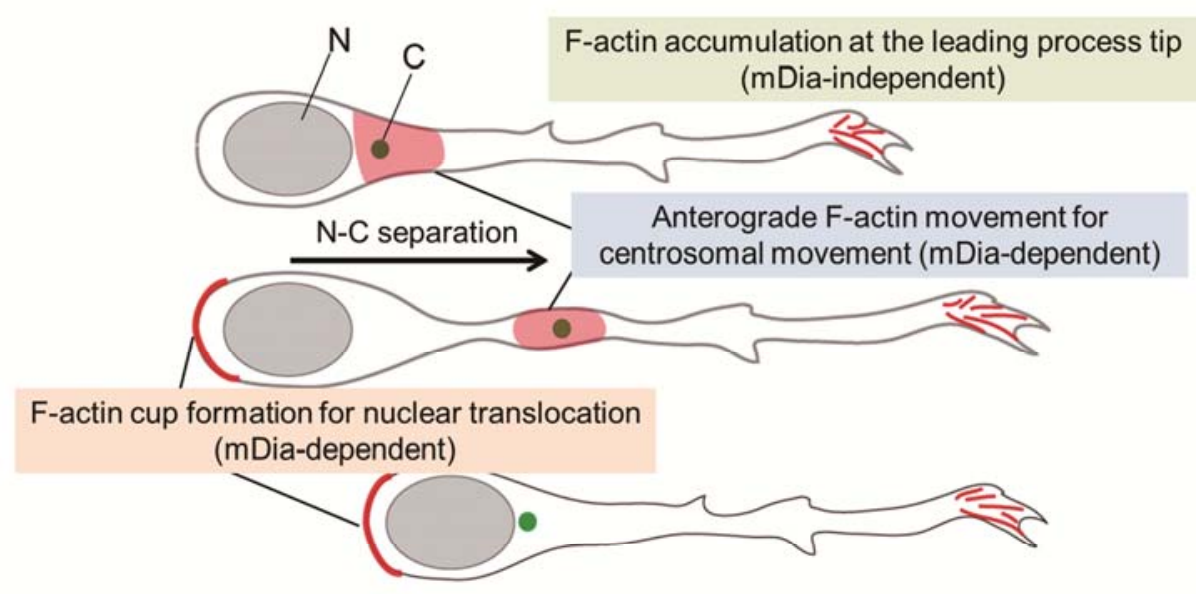

Direction of migration

Supplementary Figure 15 A proposed model of mDia functions in tangential migration.

In tangential migration, mDia mediates two types of F-actin dynamics: anterograde F-actin movement toward the distal portion of the leading process from cell body and F-actin accumulation at the rear edge of the cell. We propose that these F-actin dynamics contribute to the movement of the centrosome and the nucleus, respectively. On the other hand, mDia is not required for F-actin accumulation at the tip of the leading process. F-actin condensation and the centrosome are indicated in red and green, respectively. $\mathrm{N}$, nucleus; $\mathrm{C}$, centrosome. 


\section{REFFERENCES}

1. Watanabe, S., et al. mDia2 induces the actin scaffold for the contractile ring and stabilizes its position during cytokinesis in NIH 3T3 cells. Mol. Biol. Cell 19, 2328-2338 (2008).

2. Sakata, D., et al. Impaired T lymphocyte trafficking in mice deficient in an actin-nucleating protein, mDia1. J. Exp. Med. 204, 2031-2038 (2007). 